\title{
Global transcriptome profile of the developmental principles of in vitro iPSC-to-motor neuron differentiation
}

Emilia Solomon ${ }^{1 \dagger}$, Katie Davis-Anderson ${ }^{1 \dagger}$, Blake Hovde $^{1}$, Sofiya Micheva-Viteva ${ }^{1 *}$ (D, Jennifer Foster Harris ${ }^{1}$, Scott Twary ${ }^{1}$ and Rashi lyer ${ }^{2^{*}}$

\begin{abstract}
Background: Human induced pluripotent stem cells (iPSC) have opened new avenues for regenerative medicine. Consequently, iPSC-derived motor neurons have emerged as potentially viable therapies for spinal cord injuries and neurodegenerative disorders including Amyotrophic Lateral Sclerosis. However, direct clinical application of iPSC bears in itself the risk of tumorigenesis and other unforeseeable genetic or epigenetic abnormalities.

Results: Employing RNA-seq technology, we identified and characterized gene regulatory networks triggered by in vitro chemical reprogramming of iPSC into cells with the molecular features of motor neurons (MNs) whose function in vivo is to innervate effector organs. We present meta-transcriptome signatures of 5 cell types: iPSCs, neural stem cells, motor neuron progenitors, early motor neurons, and mature motor neurons. In strict response to the chemical stimuli, along the MN differentiation axis we observed temporal downregulation of tumor growth factor- $\beta$ signaling pathway and consistent activation of sonic hedgehog, Wnt/ $\beta$-catenin, and Notch signaling. Together with gene networks defining neuronal differentiation (neurogenin 2, microtubule-associated protein 2, Pax6, and neuropilin-1), we observed steady accumulation of motor neuron-specific regulatory genes, including Islet-1 and homeobox protein HB9. Interestingly, transcriptome profiling of the differentiation process showed that $\mathrm{Ca}^{2+}$ signaling through CAMP and LPC was downregulated during the conversion of the iPSC to neural stem cells and key regulatory gene activity of the pathway remained inhibited until later stages of motor neuron formation. Pathways shaping the neuronal development and function were well-represented in the early motor neuron cells including, neuroactive ligand-receptor interactions, axon guidance, and the cholinergic synapse formation. A notable hallmark of our in vitro motor neuron maturation in monoculture was the activation of genes encoding Gcoupled muscarinic acetylcholine receptors and downregulation of the ionotropic nicotinic acetylcholine receptors expression. We observed the formation of functional neuronal networks as spontaneous oscillations in the extracellular action potentials recorded on multi-electrode array chip after 20 days of differentiation.
\end{abstract}

(Continued on next page)

\footnotetext{
* Correspondence: sviteva@lanl.gov; rashi@lanl.gov

${ }^{\dagger}$ Emilia Solomon and Katie Davis-Anderson contributed equally to this work.

${ }^{1}$ Los Alamos National Laboratory, Bioscience Division, Los Alamos, NM, USA

${ }^{2}$ Los Alamos National Laboratory, Analytics, Intelligence, and Technology

Division, Los Alamos, NM, USA
}

(c) The Author(s). 2021 Open Access This article is licensed under a Creative Commons Attribution 4.0 International License, which permits use, sharing, adaptation, distribution and reproduction in any medium or format, as long as you give appropriate credit to the original author(s) and the source, provide a link to the Creative Commons licence, and indicate if changes were made. The images or other third party material in this article are included in the article's Creative Commons licence, unless indicated otherwise in a credit line to the material. If material is not included in the article's Creative Commons licence and your intended use is not permitted by statutory regulation or exceeds the permitted use, you will need to obtain permission directly from the copyright holder. To view a copy of this licence, visit http://creativecommons.org/licenses/by/4.0/ The Creative Commons Public Domain Dedication waiver (http://creativecommons.org/publicdomain/zero/1.0/) applies to the data made available in this article, unless otherwise stated in a credit line to the data. 
(Continued from previous page)

Conclusions: Detailed transcriptome profile of each developmental step from iPSC to motor neuron driven by chemical induction provides the guidelines to novel therapeutic approaches in the re-construction efforts of muscle innervation.

Keywords: iPSCs, Motor neurons, Stem cell reprogramming, Transcriptomics, Neuronal development, In vitro neuronal networks

\section{Background}

Human neuronal tissue lacks regenerative capacity, leaving few treatments available following neuronal injury or neurodegeneration. In the past decade, an interest in direct neuronal reprogramming of stem cells into motor neurons (MNs) has emerged as a solution to generate human neuronal tissue for therapeutic applications. MNs form synapses to potentiate electrical signals from the CNS into peripheral tissues. They play a critical role in the formation of neuromuscular junctions (NMJs), where $\mathrm{MN}$ axons terminate on muscle fibers and neurotransmitters are released to trigger muscle contractions. NMJs are cholinergic synapses, where the neurotransmitter acetylcholine (ACh) is released from the presynaptic MN terminal for uptake by postsynaptic ACh receptors on the target muscle cell [1]. This critical function is disrupted in neurodegenerative motor neurons diseases like as Amyotrophic Lateral Sclerosis (ALS).

Several in vitro protocols have been developed to convert progenitor cells, such as human inducible pluripotent stem cells (iPSCs), into MNs [2-4]. There are still challenges limiting clinical application of these iPSCderived MNs. For example, the generation of physiologically active neurons requires a lengthy cell maturation period and often results in a heterogeneous population of neuronal subtypes [5]. Protocol reproducibility can also vary as different cell lineages have unique maturation and functional properties. To address these challenges, we present a 28-day transcriptome study coupled with functional assays. Our main objective was to resolve the underlying mechanisms driving $\mathrm{MN}$ differentiation. The results from this study can guide experimental strategies to obtain populations highly enriched with the desired MN subtype.

Here, we followed an established protocol [6] to differentiate iPSCs into MNs using chemically defined media conditions. Efficient neural conversion is based on mimicking in vivo neurogenesis where extrinsic and intrinsic signals are introduced in culture, yielding a relatively pure MN population [7, 8]. During neuronal differentiation, there are cascading effects as signaling pathways activate transcription factors to upregulate expression of MN specific genes. Neural induction of iPSCs is driven by simultaneous inhibition of tumor growth factor- $\beta$ (TGF $\beta)$, activin, Nodal, and bone morphogenic protein
(BMP) signaling. Similar to processes that occur during early development, inhibition of those signaling pathways promotes differentiation along the neuronal lineage primarily through inhibition of pluripotency and blocking alternative lineage differentiation. Several other pathways, including the Wnt signaling pathway, regulate neuronal differentiation. The protocol implemented in this study included three core chemical compounds to inhibit TGF $\beta$ and BMP signaling pathways and simultaneously activate Wnt signaling. Following neural induction of iPSCs, neuronal progenitor cells were patterned with all-trans retinoic acid (RA), to promote caudal (spinal cord) identity, and ventralization was promoted by activation of Shh signaling with Purmorphamine. Finally, synchronization of the maturation process, through elimination of dividing cells, was aided by inhibition of the Notch signaling pathway resulting in mature MNs.

Genome-wide transcriptome studies provide in-depth knowledge of regulatory pathways that shape cellular morphology and function. Such information is crucial for the design of novel neuron regenerative therapies and cell-based drug discovery platforms. Previous studies based on single cell transcriptomics of Amyotrophic Lateral Sclerosis (ALS) patient-derived iPSCs discovered the underlying mechanisms of disease pathology [9] and the regulatory dynamics of $\mathrm{MN}$ differentiation [10]. Others have investigated the iPSC-derived MN axonal transcriptome and found key regulatory pathways presenting potential drug targets for treatment of genetic disorders [11]. A detailed transcriptomics study by Burke et al. proposed an influence of the genetic background of the iPSC donors on each pivotal step of iPSC-initiated corticogenesis [12]. Further, single cell RNA-seq analysis of iPSC-derived spinal MN demonstrated that in vitro differentiation does not produce a homogeneous MN population [8].

Here we performed a comprehensive transcriptomic analysis of in vitro iPSCs-derived MNs to characterize the key principles of $\mathrm{MN}$ development by analyzing bulk transcriptome data from five crucial time points of $\mathrm{MN}$ neurogenesis: iPSCs (D0), NSCs (D7), post-mitotic MNP cells (D13), eMNs (D18), and mature MNs (D28). We interrogated the transcriptomic signatures using nextgeneration RNA-sequencing (RNA-Seq) technology and 
performed in situ validation of key pluripotency and MN specific biomarker expression. Additionally, we characterized the functionality of iPSC-derived MNs via electrophysiological analysis of neuronal network connectivity. Our results corroborate transcriptomic profiles previously identified in the astrocyte-to-neuron transformation process [3]; specifically, upregulation of Shh and Wnt signaling pathways. We observed altered gene expression of TGF $\beta$ signaling components during the early stages of iPSC conversion into neural stem cell (NSC) in response to chemical inhibition. However, we detected an upregulation of positive TFG $\beta$ regulators in the subsequent steps of neurogenesis.

By applying a LANL-developed Ontology Pathway Analysis software (OPaver) we found that calcium $\left(\mathrm{Ca}^{2+}\right)$ signaling through cyclic adenosine monophosphate (cAMP) and LPC is downregulated during the conversion of the iPSC to NSC and remains silenced until the final stage of $\mathrm{MN}$ maturation when the regulatory pathway becomes a driving force for the neuronal synaptic activity. Another key finding is that during the in vitro development of $\mathrm{MN}$ in 2D monocultures, ionotropic $\mathrm{nACh}$ are expressed in the early $\mathrm{MN}$ stage and are subsequently replaced by the $\mathrm{G}$ protein coupled receptors (GPCR) -type muscarinic AChR in mature $\mathrm{MN}$, probably due to lack of metabolic stimuli that are naturally released by the muscle tissue in vivo. While our findings provide unique insights into the temporal mechanism of iPSC-derived MNs they also indicate the advantages of using a co-culture system, of $\mathrm{MN}$ and muscle, to enable the development of physiologically relevant MNs types akin to that obtained in vivo.

\section{Results}

\section{A four-step process of iPSC differentiation into MNs}

The initiating step for iPSC differentiation to neuronal stem cells (NSC) is driven by inhibition of TGF $\beta$, activin, nodal, and BMP signaling pathways while simultaneously activating the Wnt signaling pathway to sustain cell proliferation. Therefore, to convert undifferentiated iPSCs into a NSC lineage the culture media was supplemented with SB431542 (SB) and DMH-1, inhibitors of activin receptor-like kinases (ALK4, ALK5, ALK7, and BMP), and CHIR99021 (CHIR), a Wnt pathway activator. MNPs and early motor neurons (eMNs) were patterned through activation of the Shh pathway by Purmorphamine (Pur). The final step of MN maturation and specification was aided by a Notch signaling pathway inhibitor, Compound E (CpdE), to synchronize the maturation process through the elimination of dividing cells. Neurotrophic and growth factors were supplemented to facilitate neuronal growth and maturation (Fig. 1a).

Within a 13-day period, we observed changes in cell morphology, including a gradual reduction in the cell soma and multiple extensions of thin neurites upon iPSCs conversion into MNPs. Furthermore, we monitored protein accumulation of tissue-specific markers via immunohistochemistry staining (Fig. 1b). Consistent with the morphological features exemplified by high nuclear-to-cytoplasmic ratio and compact multilayer colonies [13], the undifferentiated iPSCs expressed a key pluripotency marker, Oct4 (Fig. 1b; D0). We noted the formation of NSCs at D7 by the disappearance of Oct4 and the accumulation of Nestin and Pax6. Nestin, implicated in cell division and radial axon growth [14, 15], was downregulated by D13 which coincided with increased expression of the pan-neuronal filament protein class III $\beta$-Tubulin ( $\beta$ III-Tub). Cells entered the MNP developmental stage on D13 and transformed to eMNs on D18 when both Nestin and Pax6 expression was significantly downregulated (Fig. 1b, d). Finally, we detected mature MNs featuring high levels of the structural proteins, $\beta$ III-Tub and microtubule-associated protein 2 (MAP2); and increased accumulation of MN specific markers: MN homeobox protein (HB9), choline acetyltransferase (ChAT), and the synaptic vesicles protein (Synaptophysin) (Fig. 1c, d). HB9 is an essential transcription factor and early marker of cholinergic neurons [16] and ChAT is an enzyme required for the synthesis of the neurotransmitter acetylcholine (ACh). The changes in morphology were pronounced as dendrites extended and expressed MAP2, a neuron-specific cytoskeletal protein critical for successful projection of dendrites [17, 18]. Accumulation of the pan-MN marker ISL-1 $(\sim 60 \% \pm 12 \%$, Fig. 1e) together with the HB9 transcription regulator $(\sim 85 \% \pm$ $15 \%$, Fig. 1d) at D28 of the iPSC differentiation indicated at formation of cell populations enriched in mature MNs.

\section{MNs form functional network connections}

$\mathrm{MN}$ activity was characterized by multi-electrode array (MEA) recordings of cellular electrophysiological responses. This non-invasive method allows for repetitive recordings of spontaneous electrical firings at various times during neuronal differentiation. Spontaneous oscillations in extracellular action potential (AP) were recorded after day 20 of neuronal differentiation, and the $\mathrm{AP}$ frequency increased the longer the cells differentiated on the MEA (Fig. 2a). By day 31 (D31) the firing patterns became more organized in highly synchronous bursts of network activity. The AP spike rate increased as differentiation progressed (Fig. 2b). The increase in the number of bursts, and percentage of spikes in bursts, signified successful formation of synaptic connections with synchronized AP firings (Fig. 2c). 


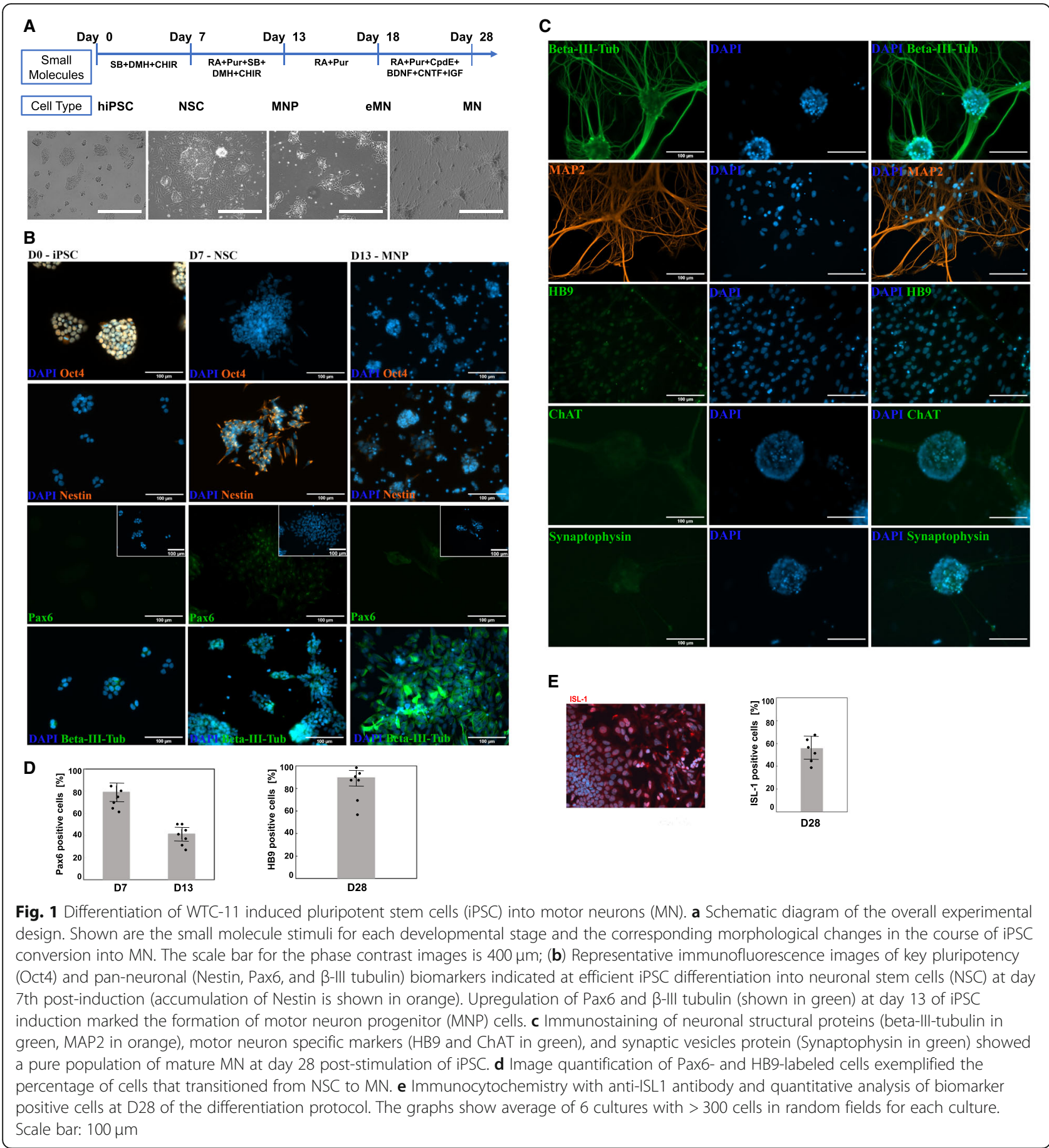

\section{Unbiased transcriptome analysis of chemically stimulated iPSC differentiation into MNs}

We performed whole-genome transcriptome analysis on RNA-Seq platform to explore gene regulatory pathways. Three independent biological replicates were collected and analyzed at each stage: iPSCs, NSCs, MNPs, eMNs, and MNs. The principal component analysis (PCA) of global transcriptome data revealed tight clustering of all replicates, indicating high reproducibility of gene expression profiles for each stage of development. Differentiation timepoints showed distinct genetic programs, as evidenced by the PCA variances (Fig. 3a). Applying pair-wise analyses, we further compared the differential gene expression profiles between cell populations at various developmental stages. The number of differentially expressed genes (DEGs) increased at each timepoint throughout the differentiation process when compared to D0, illustrating the steady transformation 


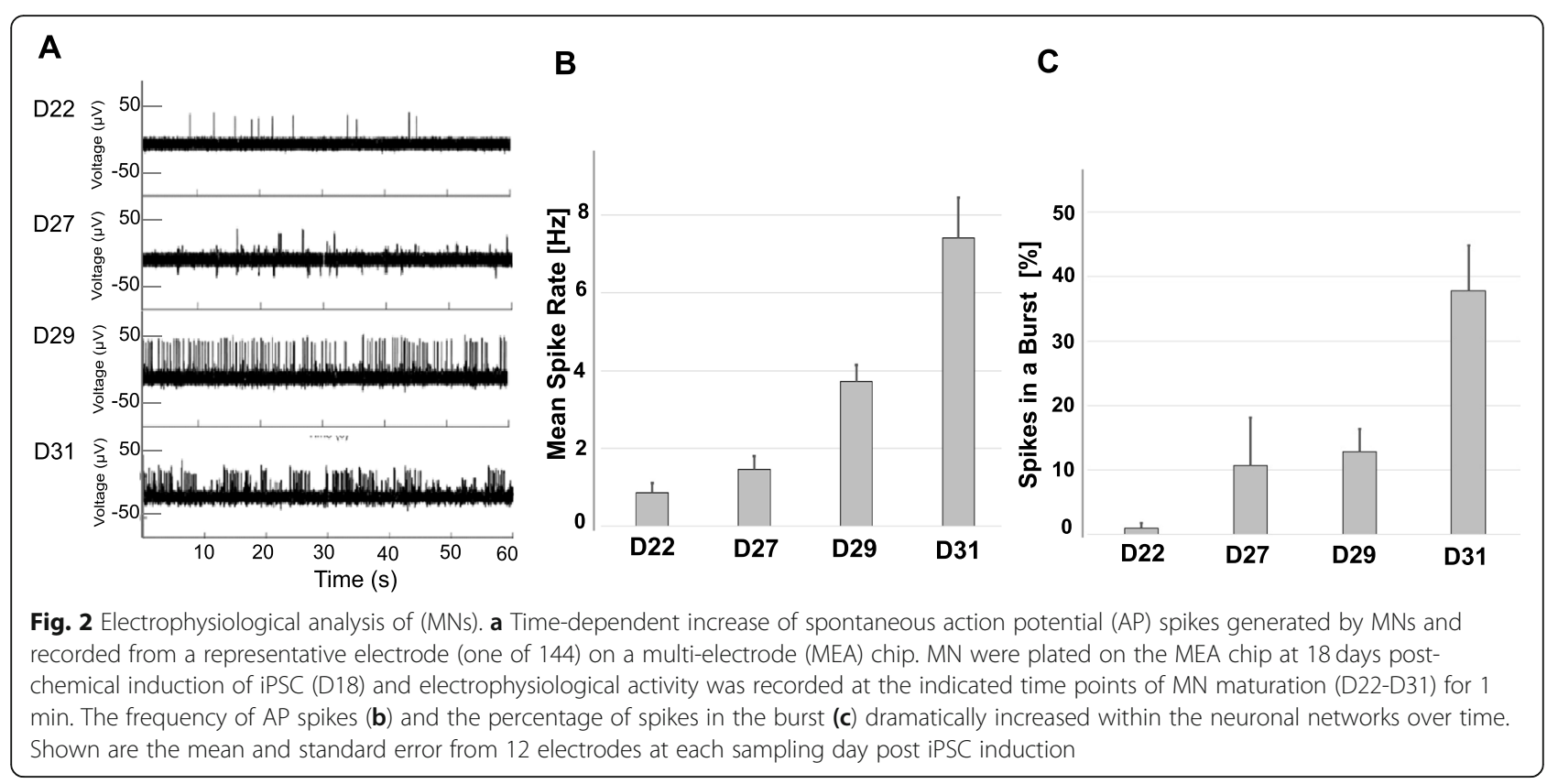

of iPSCs into MNs driven by chemical stimuli (Fig. 3b and list of DEG in Additional file 1). The greatest number of DEGs occurred upon the initial culturing of iPSCs in neural differentiation media supplemented with an inhibitor of TGF $\beta$ signaling pathway and a Wnt pathway agonist (Fig. 3c; D7-D0, see Additional file 1).

We analyzed RNA-Seq data with the NCBI Database for Annotation, Visualization and Integrated Discovery (DAVID) v6.8 [19] which categorized DEGs between D0 and D28 by gene ontology (GO) terms (Fig. 4a). Of the upregulated genes, 2118 were recognized by DAVID and 1683 (79.5\%) mapped to specific GO terms consistent with a shift from undifferentiated mitotic cells (iPSC) to differentiated cells of the neuronal lineage. The upregulated DEGs were associated with GO terms specific to neuronal development: 98 genes were related to dendrites; 144 genes were involved in cell junctions, 68 genes were associated with axon formation, and 61 genes
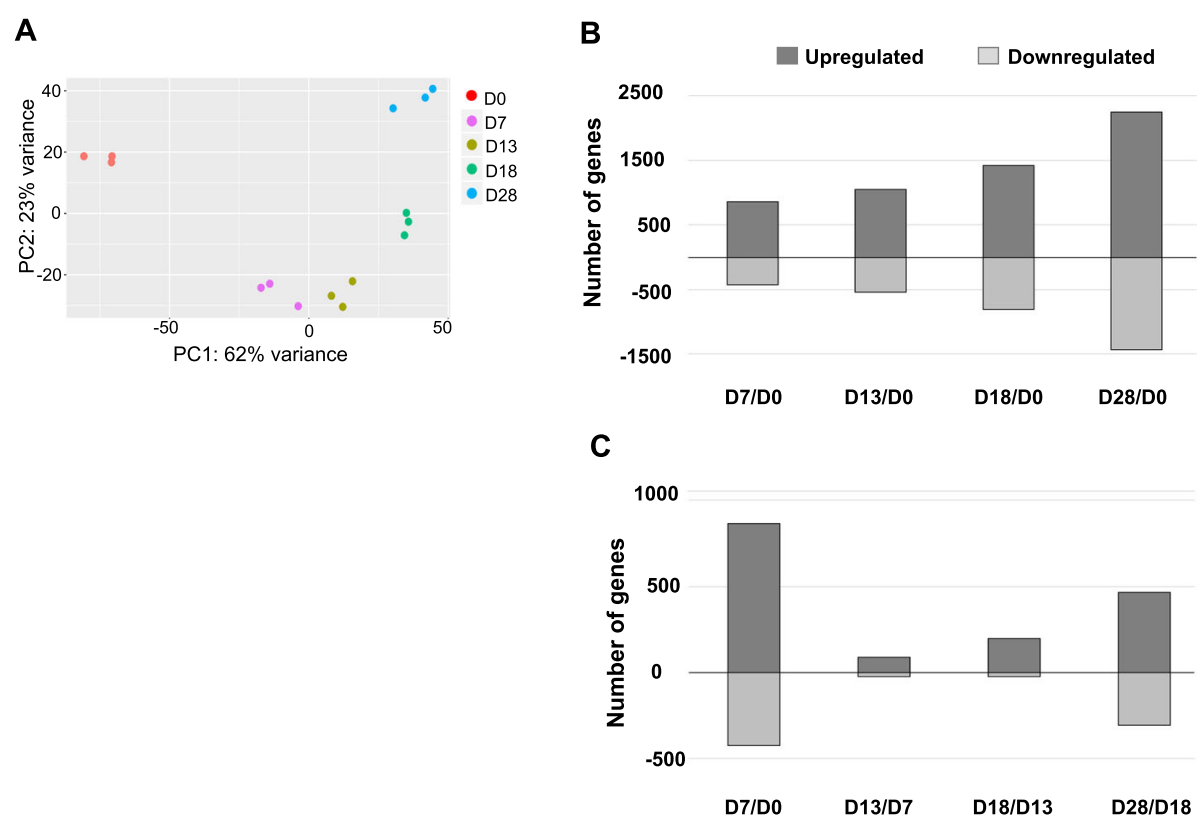

Fig. 3 Transcriptome analysis of cellular responses to MN maturation. a Principal component analysis of all samples, $n=3$; (b) Histogram of differentially expressed genes (DEGs) (adjusted $p<0.0002$, fold change $>3$ ) among D7 - D28 samples in the pairwise comparison with D0; (c) Histogram of DEGs in pairwise comparisons between adjacent timepoints (D0-D7, D7-D13, D13-18, D18-28) 

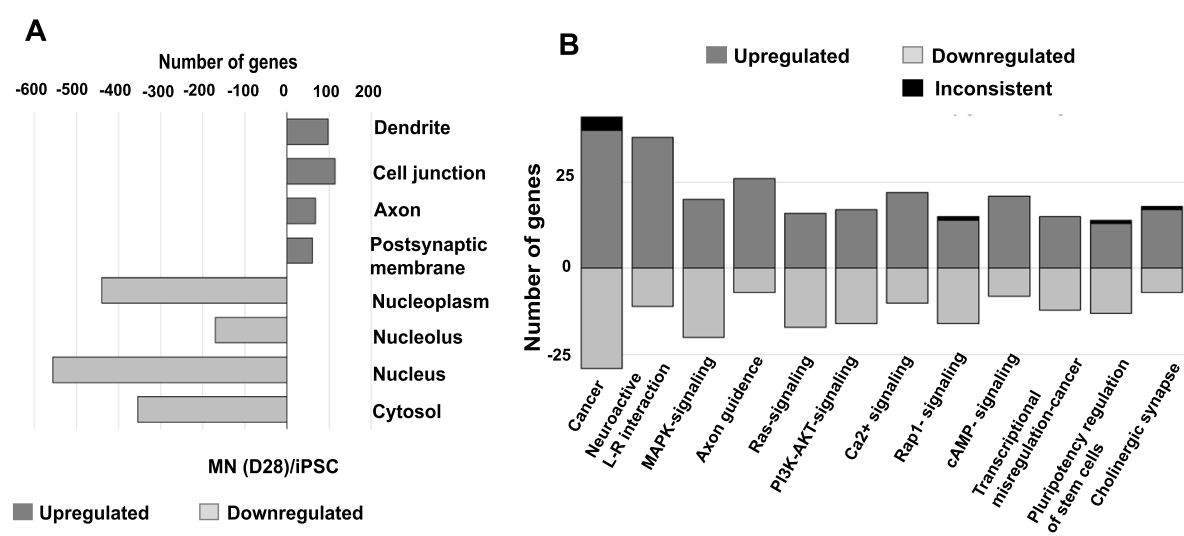

Fig. 4 Bioinformatics analysis of transcriptomic data. a Gene ontology (GO) analysis of iPSCs and MNs as determined by the NCBI Database for Annotation, Visualization and Integrated Discovery (DAVID). The top four significant GO terms of upregulated and downregulated genes are listed, ranked by $p$-value, when comparing D0 iPSCs and D28 MNs. b OPaver analysis of 12 KEGG pathways ranked by the number of DEG during the chemical reprogramming of iPSCs into MNs: Pathways in cancer; Neuroactive ligand-receptor interaction; MAPK-signaling pathway; Axon guidance; Ras signaling pathway; PI3K-Akt signaling pathway; Calcium signaling pathway; Rap1 signaling pathway; CAMP signaling pathway; Transcriptional mis-regulation in cancer; Singling pathways regulating pluripotency of stem cells; and Cholinergic synapse

were involved in the postsynaptic membrane. Of the genes downregulated on D28 versus D0, DAVID recognized 1361 genes of which 1234 (90.7\%) mapped to a specific GO. The downregulated genes were characteristic of cells undergoing active DNA replication, transcription, and translation associated with nucleoplasm (440 genes), nucleolus (170 genes), nucleus (556), and cytosol (354) GO terms (Fig. 4a).

LANL-developed OPaver [20] identified 12 gene signaling pathways that were significantly $(p<0.05)$ altered during iPSC conversion to MNs (Fig. 4b). The top 5 KEGG pathways represented genes involved in cancer regulation, axon guidance, calcium signaling, PI3K-Akt signaling, and MAPK-signaling pathways (Fig. 4b). The OPaver analysis further highlighted the critical role of membrane proteins in cell-to-cell interactions for neuronal development; specifically, neuroactive ligandreceptor interactions and the development of cholinergic synapses.

By focusing our analysis on cellular development pathways, we found significantly altered gene expression profiles of genes involved in TGF $\beta$, Notch, and Shh signaling pathways (Fig. 5). Contrary to our expectations, 7 days after chemical inhibition of TGF $\beta$, we observed a significant downregulation of genes acting as negative regulators of TGF $\beta$ signal transduction: PMEPA1 and TGIF1, repressors of SMAD2 function, and SKIL the nuclear repressor of TGF $\beta$-responsive genes [21]. At the same time, a gene positively regulated by TGF $\beta$ signaling, JUNB/AP1, was upregulated (Fig. 5a). These data indicate there was a transient inhibition (lasting less than 7 days) of the TGF $\beta$ signaling pathway, when small molecules were present in the culture media to target ALK receptor kinases. In the Notch signaling pathways, we observed a downregulation of Hes1, NOTCH2, and PRKCA after NSCs conversion into MNPs (Fig. 5b; D13). Interestingly, gene expression of Hes5 and JAG1 was upregulated until D18 when cells entered the eMN developmental stage. The final MN maturation phase required the addition of CpdE, a Notch signaling pathway inhibitor [22], along with growth factors: insulin-like growth factor 1 (IGF-1), brain-derived neurotrophic factor (BDNF), and ciliary neurotrophic factor (CNTF). Our results indicated that downregulation of Notch regulators JAG1 and Hes5 gene expression was required for the maturation of MNs (Fig. 5b; D28). Shh signaling is essential for patterning and specification of neuronal cells. Significant activation of a key Shh receptor gene, PTCH1, was observed at D7 prior to the addition of Pur, and remained upregulated throughout the differentiation process but decreased in MNs (D28), indicating that this gene may act as a positive regulator of cell division in human neuronal cells. In contrast, ARHGAP36 and CRMP1 genes were downregulated upon transition of iPSCs to NSCs (D7) and responded to Pur with gradual stimulation of gene expression in MNs at D18-28 (Fig. 5c).

\section{Validation of RNA-Seq data via RT-qPCR}

Applying RT-qPCR we further validated a subset of key gene markers of pluripotency, NSCs, MNPs, and MNs which were found to be differentially expressed by RNASeq profiling. Both methods detected the rapid decrease of pluripotency marker Oct4 by D7 and increase of Nestin, Pax6, and NgN2 expression reaching peak levels at D13 (Fig. 6a-d). Markers of MN specification (Isl1, HB9, $M A P 2$, and $C h A T$ ) increased throughout the course of neuronal maturation (Fig. 6e-f, h). ChAT expression levels gradually increased throughout the differentiation 


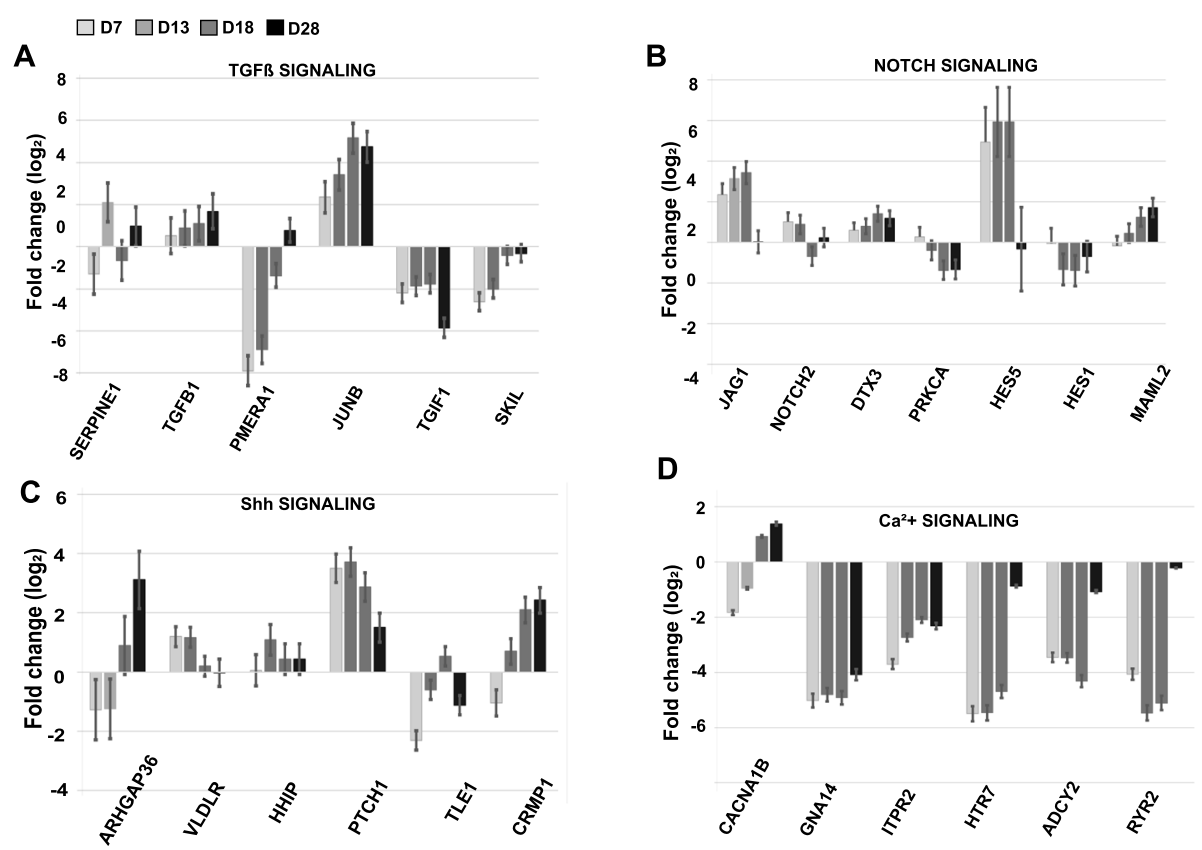

Fig. 5 Differential gene expression in key signaling pathways shaping MN differentiation. Gene transcript levels were determined by global RNAseq analysis of (a) TGF $\beta$, (b) Notch, and (c) Sonic Hedgehog (Shh) pathways and (d) Calcium signaling. Fold change was calculated relative to RNA reads in iPSC (D0). The statistics (average value and standard error) were derived from three independent biological replicas with $p<0.001$ determined by R-statistical analysis package

process from NSCs to MNs (Fig. 6h). Collectively, quantitative analysis of gene expression via RNA-Seq closely correlated with RT-qPCR data showing dynamics of gene activities typical for the conversion of pluripotent cells to MNs. We observed transient activation of Nestin, a key regulator of cytoskeletal dynamics and cell division in NSC, and stable expression of pan-neuronal markers Pax6, NgN2, and MAP2. Our data indicated there was gradual accumulation of gene transcripts responsible for the synthesis of neurotransmitter, ChAT, and transcription factors, Isl1 and $H B 9$, whose concerted actions shape the phenotype and physiological activity of MNs.

\section{Cell signaling pathways driving iPSC conversion to MNs}

The GO analysis, conducted by OPaver, identified 12 pathways that included a high number of DEGs as iPSCs were differentiated into MNs. We further investigated the DEG pattern of individual genes in the pathways listed below which had a critical role in shaping neuronal development and function (Tables 1, 2, 3, 4, 5). In each description below, 'upregulated' and 'downregulated' gene expression refers to a significance of $p<0.05$ and $\log _{2}$ fold change $\geq 2$ between sampling timepoints.

\section{MAPK-signaling pathway}

MAPK-signaling (Table 1) has an important role in mediating neuronal differentiation and survival [23]. Consistent with our expectations, the transition from iPSCs to NSCs was guided by inhibition of signal transduction pathways supporting self-renewal and activation of genes regulating neuron-specific development pathways. Gene expression of Neurotrophic Tyrosine Kinase Receptor type 2, NTRK2, was prominently upregulated at D7 when the NSC population was established, while a subset of genes encoding for $\gamma$-subunits of voltagedependent calcium-channels (Cavs) were dramatically downregulated (CACNG5, CACNG7, and CACNG8), along with $M Y C$, Ras guanyl-releasing protein 2 (RASG $R P 2$ ), and GNG12. Gene groups encoding the auxiliary subunits of high-voltage activated (HVA) Cavs were upregulated during the early stages of neuronal development [24]: $C A C N B 3$ (induced 4-fold at D7) and $C A C N$ A2D3 (16-fold activation at D7-D13). The late stage of MN formation (D18-D28) was marked by upregulation of CACNA1E and CACNG2. Several gene groups encoding auxiliary subunits of low-voltage activated (LVA) Cavs (CACNG5 and CACNG8), were initially downregulated in NSC and later upregulated during the $\mathrm{MN}$ maturation stage (D18-D28). Accumulation of Cavs gene transcripts throughout the MN differentiation process is consistent with the increased excitability of neuronal cells (Fig. 2).

Other key components of MAPK-signaling showed oscillatory patterns of gene expression throughout $\mathrm{MN}$ formation and maturation. Gene transcripts of KIT and its ligand, KITLG, which function in stem cell maintenance, were upregulated in NSCs (D7) and downregulated 


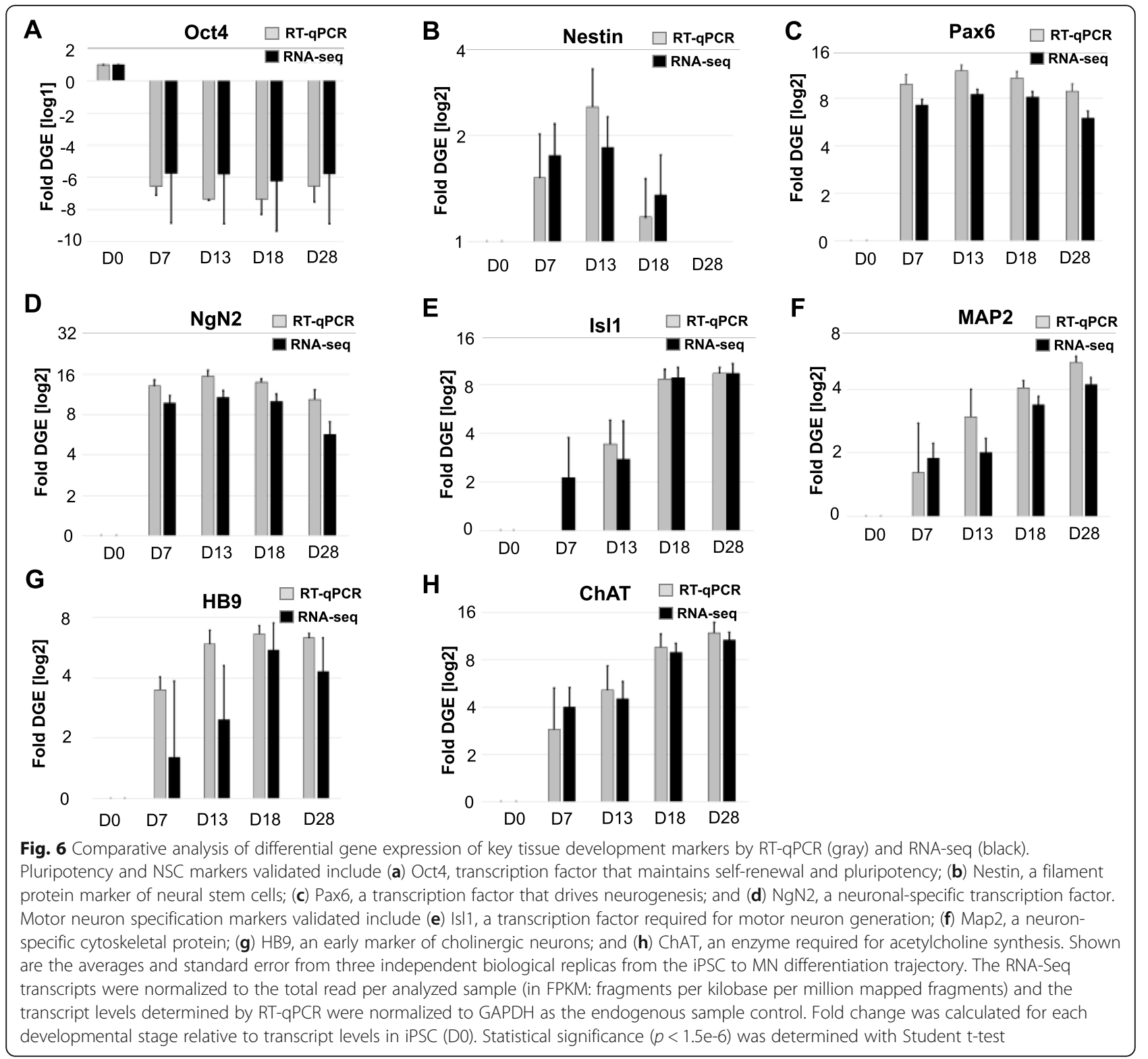

during the transition from MNP to eMN (D13-D18). KITLG transcription was again upregulated in $\mathrm{MNs}$ (D18-28). KIT signaling promotes cell survival through the activation of PIK3, PLC, and AKT1 pathways and we detected upregulation of KITLG gene expression in MNs which may be a response to the addition of IGF-1 in the culture media. Upregulation of various genes regulating cell survival and proliferation in the post-mitotic MN, such as KDR, PTPRR, PTPN5, and PRKCB, further exemplifies the MN response to IGF-1 pro-survival stimulus.

\section{Calcium $\left(\mathrm{Ca}^{2+}\right)$ signaling pathway}

$\mathrm{Ca}^{2+}$-signaling (Table 2) is a critical component of synaptic activity and neuronal function [25]. Our RNASeq data indicated the conversion of iPSCs into NSCs is marked by a significant downregulation of a gene encoding for a serotonin-specific GPCR, 5-hydroxytryptamine receptor 7 (HTR7). In addition, adenylate cyclase 2 $(A D C Y 2)$ was also downregulated at this time and is known to act immediately downstream of HTRs. Together, the downregulation of HTR7 and ADCY2 indicates a functional silencing of the cAMP-dependent signaling pathway. Furthermore, multiple genes involved in the activation of $\mathrm{Ca}^{2+}$ signaling were downregulated: GPCR subunit alpha-14 (GNA14), an activator of phospholipase C (LPC); two gene groups encoding inositol 1,4,5-triphosphate receptors (ITPR2 and ITPR3); and ryanodine receptor 2 (RYR2). (Fig. 5d and Additional File 2) One GPCR from the chemokine family, CXCR4, involved in the cytosolic $\mathrm{Ca}^{2+}$ mobilization and 
Table 1 DEGs identified in KEGG pathway: MAPK-signaling. Differential expression indicates the average log $_{2}$-fold change in RNASeq transcript levels from three independent experiments at each sampling period: D7 vs D0, D13 vs D7, D18 v D13, and D28 v D18. The $p$-values were $\leq 0.001$. Negative $\log _{2}$-fold change corresponds to gene downregulation and the positive values indicate gene activation

\begin{tabular}{|c|c|c|c|}
\hline \\
\hline \multicolumn{4}{|c|}{$\begin{array}{l}\text { MAPK-signaling pathway } \\
\text { Gene ID }\end{array}$} \\
\hline CACNA1B & voltage-dependent calcium channel $\mathrm{N}$ type alpha-1B & -1.834598101 & D0-D7 \\
\hline CACNA2D1 & voltage-dependent calcium channel alpha-2/delta-1 & -5.013073193 & D0-D7 \\
\hline CACNB3 & voltage-dependent calcium channel beta-3 & 1.96153735 & D0-D7 \\
\hline CACNG5 & voltage-dependent calcium channel gamma- 5 & -3.611690367 & D0-D7 \\
\hline CACNG7 & voltage-dependent calcium channel gamma-7 & -2.217348209 & D0-D7 \\
\hline CACNG8 & voltage-dependent calcium channel gamma-8 & -3.68477059 & D0-D7 \\
\hline FGF2 & fibroblast growth factor 2 & -1.519463128 & D0-D7 \\
\hline KITLG & KIT ligand & 2.167299479 & D0-D7 \\
\hline NTRK2 & neurotrophic tyrosine kinase receptor type 2 & 8.559108654 & D0-D7 \\
\hline KIT & proto-oncogene tyrosine-protein kinase Kit & 2.471526791 & D0-D7 \\
\hline FLT1 & MS-like tyrosine kinase 1 & -6.287270931 & D0-D7 \\
\hline $\mathrm{KDR}$ & kinase insert domain protein receptor & -5.882467261 & D0-D7 \\
\hline TGFB2 & transforming growth factor beta-2 & 2.871123317 & D0-D7 \\
\hline RASGRP2 & RAS guanyl-releasing protein 2 & -3.455335916 & D0-D7 \\
\hline GNG12 & guanine nucleotide-binding protein $\mathrm{G}(\mathrm{I}) / \mathrm{G}(\mathrm{S}) / \mathrm{G}(\mathrm{O})$ subunit gamma-12 & -2.188972021 & D0-D7 \\
\hline MYC & Myc proto-oncogene protein & -2.952930394 & D0-D7 \\
\hline MECOM & ecotropic virus integration site 1 protein & 3.315425714 & D0-D7 \\
\hline PPM1B & protein phosphatase 1B & -1.591315588 & D0-D7 \\
\hline ERBB4 & receptor tyrosine-protein kinase erbB-4 & 4.531919812 & D7-D13 \\
\hline CACNA1B & voltage-dependent calcium channel $\mathrm{N}$ type alpha-1B & 1.88048051 & D7-D13 \\
\hline CACNA2D3 & voltage-dependent calcium channel alpha-2/delta-3 & 3.068907158 & D7-D13 \\
\hline NTRK1 & neurotrophic tyrosine kinase receptor type 1 & 4.469959879 & D7-D13 \\
\hline TGFB2 & transforming growth factor beta- 2 & 2.803343326 & D7-D13 \\
\hline KIT & proto-oncogene tyrosine-protein kinase Kit & -2.467841933 & D13-D18 \\
\hline CACNG5 & voltage-dependent calcium channel gamma- 5 & 4.656489914 & D13-D18 \\
\hline FGFR4 & fibroblast growth factor receptor 4 & -3.26316459 & D13-D18 \\
\hline CACNA1E & voltage-dependent calcium channel $\mathrm{R}$ type alpha- $1 \mathrm{E}$ & 3.425325465 & D18-D28 \\
\hline CACNG2 & voltage-dependent calcium channel gamma-2 & 3.474133324 & D18-D28 \\
\hline CACNG8 & voltage-dependent calcium channel gamma-8 & 3.189789 & D18-D28 \\
\hline KITLG & KIT ligand & 2.028343818 & D18-D28 \\
\hline EGFR & epidermal growth factor receptor & -2.084730311 & D18-D28 \\
\hline FGFR1 & fibroblast growth factor receptor 1 & -2.081183963 & D18-D28 \\
\hline FGFR2 & fibroblast growth factor receptor 2 & -3.256153332 & D18-D28 \\
\hline FGFR3 & fibroblast growth factor receptor 3 & -5.009604609 & D18-D28 \\
\hline $\mathrm{KDR}$ & kinase insert domain protein receptor & 3.118215378 & D18-D28 \\
\hline IL1RAP & interleukin 1 receptor accessory protein & 3.502622162 & D18-D28 \\
\hline PRKCB & classical protein kinase $\mathrm{C}$ beta type & 1.642454101 & D18-D28 \\
\hline PTPRR & receptor-type tyrosine-protein phosphatase $\mathrm{R}$ & 2.837257876 & D18-D28 \\
\hline PTPN5 & tyrosine-protein phosphatase non-receptor type 5 & 2.472116702 & D18-D28 \\
\hline MAPKAPK2 & mitogen-activated protein kinase-activated protein kinase 2 & -1.628879059 & D18-D28 \\
\hline CDC25B & M-phase inducer phosphatase 2 & -3.052843516 & D18-D28 \\
\hline
\end{tabular}


Table 1 DEGs identified in KEGG pathway: MAPK-signaling. Differential expression indicates the average log $_{2}$-fold change in RNASeq transcript levels from three independent experiments at each sampling period: D7 vs D0, D13 vs D7, D18 v D13, and D28 v D18. The $p$-values were $\leq 0.001$. Negative $\log _{2}$-fold change corresponds to gene downregulation and the positive values indicate gene activation (Continued)

\begin{tabular}{llll}
\hline MAPK-signaling pathway & & \\
\hline Gene ID & Description & Differential expression & Time \\
\hline MAP3K5 & mitogen-activated protein kinase kinase kinase 5 & 1.972512785 & D18-D28 \\
\hline
\end{tabular}

Table 2 DEGs identified in KEGG pathway: Calcium signaling. Differential expression indicates the average log 2 -fold change in RNASeq transcript levels from three independent experiments at each sampling period: D7 vs D0, D13 vs D7, D18 v D13, and D28 v D18. The $p$-values were $\leq 0.001$. Negative $\log _{2}$-fold change corresponds to gene downregulation and the positive values indicate gene activation

\begin{tabular}{|c|c|c|c|}
\hline \multicolumn{4}{|c|}{ Calcium signaling pathway } \\
\hline Gene ID & Description & Differential expression & Time \\
\hline HTR7 & 5-hydroxytryptamine receptor 7 & -5.492892685 & D0-D7 \\
\hline CACNA1B & voltage-dependent calcium channel N type alpha-1B & -1.834598101 & D0-D7 \\
\hline CXCR4 & C-X-C chemokine receptor type 4 & 4.604478969 & D0-D7 \\
\hline $\mathrm{ADCY} 2$ & adenylate cyclase 2 & -3.460253861 & D0-D7 \\
\hline STIM1 & stromal interaction molecule 1 & 1.834422349 & D0-D7 \\
\hline VDAC1 & voltage-dependent anion channel protein 1 & -1.32731076 & D0-D7 \\
\hline GNA14 & guanine nucleotide-binding protein subunit alpha-14 & -5.014275635 & D0-D7 \\
\hline TRDN & Triadin & -4.320486512 & D0-D7 \\
\hline RYR2 & ryanodine receptor 2 & -4.07053211 & D0-D7 \\
\hline RYR3 & ryanodine receptor 3 & 1.71133494 & D0-D7 \\
\hline ITPR2 & inositol 1,4,5-triphosphate receptor type 2 & -3.699823272 & D0-D7 \\
\hline ITPR3 & inositol 1,4,5-triphosphate receptor type 3 & -2.406497059 & D0-D7 \\
\hline ERBB4 & receptor tyrosine-protein kinase erbB-4 & 4.531919812 & D7-D13 \\
\hline CACNA1B & voltage-dependent calcium channel $\mathrm{N}$ type alpha-1B & 1.88048051 & D13-D18 \\
\hline CACNA1E & voltage-dependent calcium channel $\mathrm{R}$ type alpha- $1 \mathrm{E}$ & 3.425325465 & D13-D18 \\
\hline GRIN2A & glutamate receptor ionotropic, NMDA 2A & 2.854753149 & D13-D18 \\
\hline $\mathrm{P} 2 \mathrm{R} \times 3$ & $\mathrm{P} 2 \mathrm{X}$ purinoceptor 3 & 2.552456853 & D13-D18 \\
\hline CAMK4 & calcium/calmodulin-dependent protein kinase IV & 1.41910402 & D13-D18 \\
\hline CHRM3 & muscarinic acetylcholine receptor M3 & 3.978819163 & D18-D28 \\
\hline HTR7 & 5-hydroxytryptamine receptor 7 & 3.808433488 & D18-D28 \\
\hline GRIN1 & glutamate receptor ionotropic, NMDA 1 & 3.726336735 & D18-D28 \\
\hline GRIN2D & glutamate receptor ionotropic, NMDA 2D & 2.087932817 & D18-D28 \\
\hline CHRM2 & muscarinic acetylcholine receptor $\mathrm{M} 2$ & 3.383752478 & D18-D28 \\
\hline ADRA1A & adrenergic receptor alpha- $1 \mathrm{~A}$ & 4.207285974 & D18-D28 \\
\hline TACR3 & tachykinin receptor 3 & 2.111829478 & D18-D28 \\
\hline GRM1 & metabotropic glutamate receptor 1 & 3.134222868 & D18-D28 \\
\hline EGFR & epidermal growth factor receptor & -2.084730311 & D18-D28 \\
\hline GNAL & guanine nucleotide-binding protein $\mathrm{G}(\mathrm{olf})$ subunit alpha & 1.685044809 & D18-D28 \\
\hline $\mathrm{ADCY} 2$ & adenylate cyclase 2 & 3.240853085 & D18-D28 \\
\hline STIM2 & stromal interaction molecule 2 & 1.158068159 & D18-D28 \\
\hline RYR2 & ryanodine receptor 2 & 4.896050853 & D18-D28 \\
\hline PRKCB & classical protein kinase $\mathrm{C}$ beta type & 1.642454101 & D18-D28 \\
\hline
\end{tabular}


Table 3 DEGs identified in KEGG pathway: Neuroactive ligand-receptor interaction. Differential expression indicates the average $\log _{2}$-fold change in RNA-Seq transcript levels from three independent experiments at each sampling period: D7 vs D0, D13 vs D7, D18 $\vee$ D13, and D28 $\vee$ D18. The $p$-values were $\leq 0.001$. Negative $\log _{2}$-fold change corresponds to gene downregulation and the positive values indicate gene activation

\begin{tabular}{|c|c|c|c|}
\hline \\
\hline \multicolumn{4}{|c|}{ 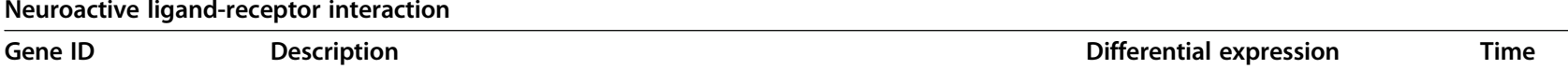 } \\
\hline APLNR & apelin receptor & 4.656030708 & D0-D7 \\
\hline NMUR2 & neuromedin $U$ receptor 2 & 8.490690944 & D0-D7 \\
\hline F2 & coagulation factor II (thrombin) & 2.589060962 & D0-D7 \\
\hline CNR1 & cannabinoid receptor 1 & 2.81817017 & D0-D7 \\
\hline S1PR1 & sphingosine 1-phosphate receptor 1 & 5.38497247 & D0-D7 \\
\hline GRM4 & metabotropic glutamate receptor 4 & -3.861684955 & D0-D7 \\
\hline GRIN2B & glutamate receptor ionotropic, NMDA 2B & -2.895136106 & D0-D7 \\
\hline CHRNA3 & nicotinic acetylcholine receptor alpha-3 & 2.7938378 & D0-D7 \\
\hline HTR7 & 5-hydroxytryptamine receptor 7 & -5.492892685 & D0-D7 \\
\hline CHRNB4 & nicotinic acetylcholine receptor beta- 4 & 4.925250798 & D0-D7 \\
\hline GRIA1 & glutamate receptor 1 & 3.01762919 & D0-D7 \\
\hline GRIA4 & glutamate receptor 4 & -2.647220311 & D0-D7 \\
\hline GRIK1 & glutamate receptor, ionotropic 42ainite 1 & 2.276415318 & D0-D7 \\
\hline GRIK4 & glutamate receptor, ionotropic 42ainite 4 & -3.020412525 & D0-D7 \\
\hline GRID2 & glutamate receptor delta-2 subunit & -5.40793957 & D0-D7 \\
\hline GLRA2 & glycine receptor alpha-2 & 1.792820308 & D0-D7 \\
\hline GLRB & glycine receptor beta & 3.452103236 & D0-D7 \\
\hline $\mathrm{NR3C1}$ & glucocorticoid receptor & -2.452514385 & D0-D7 \\
\hline GHR & growth hormone receptor & 2.976564346 & D0-D7 \\
\hline CNR1 & cannabinoid receptor 1 & 2.626395057 & D7-D13 \\
\hline NR3C1 & glucocorticoid receptor & 3.341402421 & D7-D13 \\
\hline GRIN2A & glutamate receptor ionotropic, NMDA 2A & 2.854753149 & D13-D18 \\
\hline CHRNA4 & nicotinic acetylcholine receptor alpha-4 & 2.830329136 & D13-D18 \\
\hline $\mathrm{P} 2 \mathrm{R} \times 3$ & P2X purinoceptor 3 & 2.552456853 & D13-D18 \\
\hline GRIA2 & glutamate receptor 2 & 3.273675604 & D13-D18 \\
\hline GRIA4 & glutamate receptor 4 & 2.416455621 & D13-D18 \\
\hline ADCYAP1R1 & pituitary adenylate cyclase-activating polypeptide type I receptor & 2.615605165 & D13-D18 \\
\hline CHRM2 & muscarinic acetylcholine receptor M2 & 3.383752478 & D18-D28 \\
\hline CHRM3 & muscarinic acetylcholine receptor M3 & 3.978819163 & D18-D28 \\
\hline ADRA1A & adrenergic receptor alpha- $1 \mathrm{~A}$ & 4.207285974 & D18-D28 \\
\hline DRD2 & dopamine receptor D2 & -2.728065758 & D18-D28 \\
\hline HTR7 & 5-hydroxytryptamine receptor 7 & 3.808433488 & D18-D28 \\
\hline NMU & neuromedin $U$ & -2.62455439 & D18-D28 \\
\hline NPY5R & neuropeptide $Y$ receptor type 5 & 3.804748523 & D18-D28 \\
\hline HCRTR2 & hypocretin (orexin) receptor 2 & 3.98239527 & D18-D28 \\
\hline SSTR1 & somatostatin receptor 1 & 6.807432954 & D18-D28 \\
\hline TAC1 & tachykinin 1 & 3.869735499 & D18-D28 \\
\hline TACR3 & tachykinin receptor 3 & 2.111829478 & D18-D28 \\
\hline PTGER4 & prostaglandin E receptor 4 & 2.824782168 & D18-D28 \\
\hline GPR50 & G protein-coupled receptor 50 & 4.747091061 & D18-D28 \\
\hline LPAR1 & lysophosphatidic acid receptor 1 & 1.500777066 & D18-D28 \\
\hline
\end{tabular}


Table 3 DEGs identified in KEGG pathway: Neuroactive ligand-receptor interaction. Differential expression indicates the average $\log _{2}$-fold change in RNA-Seq transcript levels from three independent experiments at each sampling period: D7 vs D0, D13 vs D7, D18 v D13, and D28 v D18. The $p$-values were $\leq 0.001$. Negative $\log _{2}$-fold change corresponds to gene downregulation and the positive values indicate gene activation (Continued)

\begin{tabular}{llll}
\hline Neuroactive ligand-receptor interaction & & \\
\hline Gene ID & Description & Differential expression & Time \\
\hline S1PR1 & sphingosine 1-phosphate receptor 1 & 2.506524278 & D18-D28 \\
ADCYAP1R1 & pituitary adenylate cyclase-activating polypeptide type I receptor & -2.231972171 & D18-D28 \\
GRM1 & metabotropic glutamate receptor 1 & 3.134222868 & D18-D28 \\
GRIN1 & glutamate receptor ionotropic, NMDA 1 & 3.726336735 & D18-D28 \\
GRIN2D & glutamate receptor ionotropic, NMDA 2D & 2.087932817 & D18-D28 \\
GRIN3A & glutamate receptor ionotropic, NMDA 3A & 2.914543821 & D18-D28 \\
CHRNA3 & nicotinic acetylcholine receptor alpha-3 & -2.670435709 & D18-D28 \\
GRIA2 & glutamate receptor 2 & 2.698334339 & D18-D28 \\
GRIA4 & glutamate receptor 4 & 1.923861676 & D18-D28 \\
GRID2 & glutamate receptor delta-2 subunit & 4.217720694 & D18-D28 \\
GRIN2B & glutamate receptor ionotropic, NMDA 2B & 4.109294839 & D18-D28 \\
GLRA3 & glycine receptor alpha-3 & 5.577349539 & D18-D28 \\
GLRB & glycine receptor beta & 1.392250382 & D18-D28 \\
\hline
\end{tabular}

cell migration, was also markedly upregulated (16-fold) during the transition into NSCs.

A hallmark of the NSC to MNP transition was the accumulation of receptor tyrosine-protein kinase erB-4 (ERBB4) transcripts. ERBB4 is activated by epidermal growth factor proteins, neuregulins $2 / 3$, to shape the development of neuronal cells through activation of MAPK3/ERK [26, 27]. ERBB4 expression was upregulated by 18 -fold in D7-D13, followed by upregulation of CACNA1B, CACNA1E, GRIN2A and P2RX3 (D13-D18). Formation of eMNs (D13-D18) coincided with upregulated gene expression of HVA $\mathrm{N}$ - and R-type (CACN $A 1 B$ and $C A C N A 1 E$ ) voltage-dependent $\mathrm{Ca}^{2+}$ channels, glutamate ionotropic receptor (GRIN2A), and the ligandgated ion channel responsible for peripheral pain responses, $P 2 R X 3$. These gene expression profiles indicate the selective pressure of chemical stimuli lead to the formation of eMNs with characteristics of the sympathetic nerve system [28].

In the final stage of $\mathrm{MN}$ maturation (D18-D28), we observed upregulation of gene groups activating $\mathrm{Ca}^{2+}$ signaling through the cAMP-dependent pathway: HTR7 and $A D C Y 2$. We also detected upregulated expression of the intracellular $\mathrm{Ca}^{2+}$ ryanodine receptor $2(R Y R 2)$, signifying cell readiness to release $\mathrm{Ca}^{2+}$ from the sarcoplasmic reticulum in response to adrenergic (sympathetic) stimulation (Fig. 5d). This finding was consistent with the upregulation of $A D R A 1 A$, which encodes the adrenergic receptor alpha subunit $1 \alpha$. Furthermore, gene activation of both ionotropic (GRIN1 and GRIN2D) and metabotropic (GRM1) glutamate receptors, together with the upregulation of neuromedin- $\mathrm{K}$ receptor (TACR3), accentuated the role of the IP3- $\mathrm{Ca}^{2+}$ second messenger system to activate ERK1/2 as well as classical PKC signaling pathways [29-31]. We also detected higher levels of $P R K C B$ gene transcripts in MNs compared to eMNs (Table 2).

Interestingly, G-coupled muscarinic acetylcholine receptors (mAChRs) were upregulated in mature MNs (D28). Genes encoding for excitatory (CHRM3) and inhibitory (CHRM2) mAChRs were specifically activated in late stage $\mathrm{MN}$ maturation (Table 2, D18-D28). The excitatory mAChRs stimulate PLC, triggering IP3 and diacylglycerol signaling pathways (Additional file 2). Since mAChRs are present on parasympathetic neurons [32], our data suggest that the iPSC-induced MNs bear the excitatory characteristics of parasympathetic neurons.

\section{Neuroactive ligand-receptor interactions}

The majority of neuronal receptors are GPCRs and they function to regulate signal transduction pathways and shape cellular physiological responses. Our genome wide transcriptome analysis revealed that iPSC-induced MNs differentiation is marked by the expression of neuroactive receptors which are responsive to glutamate, $\mathrm{ACh}$, and catecholamines, orexin, and prostaglandins (Table 3).

During the first step of iPSC commitment to a neuronal lineage, multiple glutamate receptors were altered, notably, gene expression levels of two ionotropic glutamate receptors were upregulated in the NSCs, GRIA1 and GRIK1, while their paralog genes, GRIA4 and GRIK4, were downregulated. This suggests a specific 
Table 4 DEGs identified in KEGG pathway: Axon guidance. Differential expression indicates the average log 2 -fold change in RNA-Seq transcript levels from three independent experiments at each sampling period: D7 vs D0, D13 vs D7, D18 v D13, and D28 v D18. The $p$-values were $\leq 0.001$. Negative $\log _{2}$-fold change corresponds to gene downregulation and the positive values indicate gene activation

\begin{tabular}{|c|c|c|c|}
\hline \multicolumn{4}{|c|}{ Axon guidance } \\
\hline Gene ID & Description & Differential expression & Time \\
\hline$\overline{\text { NTNG1 }}$ & netrin-G1 & -2.536297436 & D0-D7 \\
\hline DCC & deleted in colorectal carcinoma & 2.502983549 & D0-D7 \\
\hline NTN1 & netrin 1 & 3.198672486 & D0-D7 \\
\hline FYN & tyrosine-protein kinase Fyn & 1.772436052 & D0-D7 \\
\hline RGMA & repulsive guidance molecule A & 3.097407276 & D0-D7 \\
\hline CXCR4 & C-X-C chemokine receptor type 4 & 4.604478969 & D0-D7 \\
\hline CXCL12 & C-X-C motif chemokine 12 & -4.220104913 & D0-D7 \\
\hline EPHA3 & Eph receptor A3 & 3.43500774 & D0-D7 \\
\hline EPHA4 & Eph receptor A4 & 1.860959561 & D0-D7 \\
\hline EPHA7 & EphA7 & 2.088277014 & D0-D7 \\
\hline EPHB2 & Eph receptor B2 & 1.873701283 & D0-D7 \\
\hline EPHB3 & Eph receptor B3 & 5.416063376 & D0-D7 \\
\hline ENAH & Enabled & -0.89497636 & D0-D7 \\
\hline SLIT1 & slit 1 & 5.447826267 & D0-D7 \\
\hline $\mathrm{ROBO} 2$ & roundabout, axon guidance receptor 2 & -2.472827128 & D0-D7 \\
\hline NRP1 & neuropilin 1 & 4.117109594 & D0-D7 \\
\hline DPYSL5 & dihydropyrimidinase-like 5 & 4.921620042 & D0-D7 \\
\hline PTCH1 & patched 1 & 3.517640353 & D0-D7 \\
\hline $\mathrm{BOC}$ & brother of $\mathrm{CDO}$ & 4.811514207 & D0-D7 \\
\hline BMP7 & bone morphogenetic protein 7 & 3.232399315 & D0-D7 \\
\hline BMPR1B & bone morphogenetic protein receptor type-1B & 1.78373296 & D0-D7 \\
\hline DCC & deleted in colorectal carcinoma & 2.626762708 & D7-D13 \\
\hline EPHA3 & Eph receptor A3 & 2.836614823 & D7-D13 \\
\hline PAK3 & p21-activated kinase 3 & 1.64575883 & D13-D18 \\
\hline NTNG1 & netrin-G1 & 2.991377261 & D13-D18 \\
\hline EPHA5 & Eph receptor A5 & 3.15278798 & D13-D18 \\
\hline SLIT1 & slit 1 & 2.183813571 & D13-D18 \\
\hline SLIT2 & slit 2 & 2.342825102 & D13-D18 \\
\hline NTNG1 & netrin-G1 & 2.430765649 & D18-D28 \\
\hline TRPC5 & transient receptor potential cation channel subfamily $\mathrm{C}$ member 5 & 2.464006156 & D18-D28 \\
\hline RGS3 & regulator of G-protein signaling 3 & -1.814236062 & D18-D28 \\
\hline CXCL12 & C-X-C motif chemokine 12 & 3.751564688 & D18-D28 \\
\hline EPHA5 & Eph receptor A5 & 3.524159164 & D18-D28 \\
\hline EPHA6 & Eph receptor A6 & 2.585950072 & D18-D28 \\
\hline $\mathrm{BOC}$ & brother of $\mathrm{CDO}$ & -1.858286661 & D18-D28 \\
\hline WNT4 & wingless-type MMTV integration site family, member 4 & 2.873234122 & D18-D28 \\
\hline
\end{tabular}

requirement for type 1 ionotropic glutamate receptors early in neuronal development. Neuromedin and apelin receptors (NMUR2 and APLNR) were upregulated in NSCs, as well as GPCR response to sphingosine-1phosphate signaling receptor (S1PR1), suggesting changes in cytoskeleton dynamics and mitosis [33]. Genes encoding for nicotinic acetylcholine receptors (nAChR) and cannabinoid receptors (CNR1and CHRNB4) were upregulated during the conversion of NSCs into MNPs and remained activated until eMNs were formed. 
Table 5 DEGs identified in KEGG pathway: Cholinergic synapse. Differential expression indicates the average log $_{2}-f_{0}$ ld change in RNA-Seq transcript levels from three independent experiments at each sampling period: D7 vs D0, D13 vs D7, D18 v D13, and D28 v D18. The $p$-values were $\leq 0.001$. Negative $\log _{2}$-fold change corresponds to gene downregulation and the positive values indicate gene activation

\begin{tabular}{|c|c|c|c|}
\hline \multicolumn{4}{|c|}{ Cholinergic synapse } \\
\hline Gene ID & Description & Differential expression & Time \\
\hline GNG12 & guanine nucleotide-binding protein $\mathrm{G}(\mathrm{I}) / \mathrm{G}(\mathrm{S}) / \mathrm{G}(\mathrm{O})$ subunit gamma-12 & -2.18897 & D0-D7 \\
\hline CACNA1B & voltage-dependent calcium channel $\mathrm{N}$ type alpha-1B & -1.8346 & D0-D7 \\
\hline KCNQ3 & potassium voltage-gated channel KQT-like subfamily member 3 & -2.96129 & D0-D7 \\
\hline KCNJ12 & potassium inwardly-rectifying channel subfamily J member 12 & 3.350697 & D0-D7 \\
\hline CHRNA3 & nicotinic acetylcholine receptor alpha-3 & 2.793838 & D0-D7 \\
\hline CHRNB4 & nicotinic acetylcholine receptor beta-4 & 4.925251 & D0-D7 \\
\hline ITPR2 & inositol 1,4,5-triphosphate receptor type 2 & -3.69982 & D0-D7 \\
\hline ITPR3 & inositol 1,4,5-triphosphate receptor type 3 & -2.4065 & D0-D7 \\
\hline ADCY2 & adenylate cyclase 2 & -3.4602539 & D0-D7 \\
\hline ADCY6 & adenylate cyclase 6 & 1.892735 & D0-D7 \\
\hline CREB5 & cyclic AMP-responsive element-binding protein 5 & 2.033579 & D0-D7 \\
\hline FYN & tyrosine-protein kinase Fyn & 1.772436 & D0-D7 \\
\hline CAMK4 & calcium/calmodulin-dependent protein kinase IV & 1.419104 & D7-D13 \\
\hline ChAT & choline O-acetyltransferase & 4.392642 & D13-D18 \\
\hline CACNA1B & voltage-dependent calcium channel N type alpha-1B & 1.880481 & D13-D18 \\
\hline SLC5A7 & solute carrier family 5 (high affinity choline transporter), member 7 & 4.94003258 & D13-D18 \\
\hline KCNQ2 & potassium voltage-gated channel KQT-like subfamily member 2 & 3.013059 & D13-D18 \\
\hline CHRNA4 & nicotinic acetylcholine receptor alpha-4 & 2.830329 & D13-D18 \\
\hline CHRM3 & muscarinic acetylcholine receptor $\mathrm{M} 3$ & 3.97881916 & D18-D28 \\
\hline ChAT & choline O-acetyltransferase & 1.799457 & D18-D28 \\
\hline ACHE & Acetylcholinesterase & 2.982588 & D18-D28 \\
\hline CHRM2 & muscarinic acetylcholine receptor $\mathrm{M} 2$ & 3.383752 & D18-D28 \\
\hline SLC5A7 & solute carrier family 5 (high affinity choline transporter), member 7 & 2.363264 & D18-D28 \\
\hline KCNQ3 & potassium voltage-gated channel KQT-like subfamily member 3 & 3.701006 & D18-D28 \\
\hline CHRNA3 & nicotinic acetylcholine receptor alpha-3 & -2.67044 & D18-D28 \\
\hline PRKCB & classical protein kinase $\mathrm{C}$ beta type & 1.642454 & D18-D28 \\
\hline $\mathrm{ADCY} 2$ & adenylate cyclase 2 & 3.240853 & D18-D28 \\
\hline
\end{tabular}

Formation of eMNs was marked by reactivation of genes encoding glutamate receptors which were downregulated at the NSC stage. Gene activity of various ionotropic glutamate receptors peaked when eMNs became mature MNs (D18-D28). Activation of genes regulating the synthesis of various neurotransmitters defined the transition from eMNs to MNs. The transcript levels of the neuropeptide tachykinin, and its receptor (TACR3), were upregulated in MNs. In spinal neurons, tachykinins evoke synthesis and release of ACh, histamine, catecholamines, and GABA. We also detected differential expression of the somatostatin receptor (SSTR1) indicating that the mature MN population can secrete neurotransmitters [34]. The glucocorticoid receptor (NR3C1) was upregulated in MNPs and MNs and two new classes of neuroactive receptors were upregulated in the last stage of $\mathrm{MN}$ maturation: hypocretin/orexin (HCRTR2) and prostaglandin E receptor 4 (PTGER4). Our transcriptomic data indicate that the replacement of ionotropic nAChR (CHRNA3) and dopamine receptor (DRD2) expression with adrenergic receptor (ADRA1A) and mAChRs (CHRM2 and CHRM3) marked the formation of mature MNs. The controversial activation of mAChRs and genes encoding excitatory neurotransmitters, including glutamate and catecholamines (ADRA1A), could stem from the formation of a heterogeneous population consisting of parasympathetic and sympathetic MNs. 


\section{Axon guidance and cholinergic synapse}

Pathways regulating axon guidance and synapse development were upregulated during iPSC-to-MN differentiation (Tables 4 and 5 , respectively). These functions are critical for neuronal development, maintenance, and repair mechanisms.

We found netrin-G1 (NTNG1) to be initially downregulated as iPSCs transitioned into NSC, but then upregulated in eMNs and MNs from D13-28. Deleted in colorectal carcinoma (DCC) was also significantly upregulated as iPSCs transition through NSCs to become MNPs, indicating its critical role in prompting axon extension. SLIT1 was upregulated at D7 and both SLIT1 and SLIT2 were upregulated as the NSCs became MNPs (D7-D13). HB9 tightly regulates Robo2 expression to regulate motor axon guidance in ventrally-projecting MNs [35] and we observed $\mathrm{ROBO} 2$ downregulation in NSCs, during which time HB9 transcripts were initially accumulating (Fig. 6g).

In vivo as $\mathrm{MN}$ axons extend, they have the potential to terminate on a muscle fiber at a cholinergic synapse, the site of ACh neurotransmission. In the pre-synaptic neuron, ACh synthesis is driven by choline Oacetyltransferase (ChAT) and we observed ChAT gene expression to be increased in both eMNs and MNs. Expression of ion-channel-coupled nAChR subunits were also significantly altered: CHRNA3 was regulated in NSCs but downregulated in MNs and CHRNA4 was upregulated at the eMN stage. In the postsynaptic membrane these receptor components would contribute to neuroactive ligand-receptor interactions as previously described. Gene expression of acetylcholinesterase $(A C h E)$ was significantly increased late in MNs maturation (D18-D28), which encodes an enzyme responsible for the hydrolysis of ACh into choline and acetic acid, a critical step in ACh recycling in the cholinergic synapse. Membrane transporter solute carrier family 5 member 7 (SLC5A7) is then able to import choline back into a cholinergic neuron for subsequent $\mathrm{ACh}$ synthesis. We found gene expression of $S L C 5 A 7$ to be increased in both the eMNs and mature MNs.

\section{Heterogeneity of $\mathrm{MN}$ populations}

As previously reported [8], in vitro differentiation of human iPSC resulted in heterogeneous populations consisting of several MN subtypes. Applying the same differentiation protocol [6] and quantitative image analysis (Fig. 1d-e), we observed that two pan-MN transcription regulators ISL-1and HB9 were present in $\sim 60( \pm 12 \%)$ and $\sim 85( \pm 15 \%)$ of the cell populations, respectively (Fig. 1d-e). Thus, our quantitative image analysis data is in agreement with the $68 \pm 7 \%$ double positive $\mathrm{ISL}^{+} / \mathrm{HB}^{+} \mathrm{MN}$ populations reported by Thiry et al [8]. Based on our image analysis on single cell level
(Fig. 1) and quantitative RT-PCR of bulk transcriptome (Fig. 6) we could infer that iPSC-derived neuronal populations are highly enriched on $\mathrm{MN}$ in general although without detailed analysis of the molecular markers characteristic to each $\mathrm{MN}$ subtype we are not able to conclude which one is predominant. Combination of transcription master regulators' activity determines the MN functional specifications (Table 6). Based on the quantitative image analysis, majority of the MNs were positive for the HB9 gene (Fig. 1c-d), which remains activated in mature MNs from the median and hypaxial motor columns innervating the long muscles of the back and the respiratory muscles, respectively, while HB9 is downregulated in the medial neurons of the lateral motor column (LMC) innervating the limb muscles. The meta-transcriptome data from our study showed significant $(p<1 \mathrm{e}-5)$ activation $\left(\log _{2}>2\right)$ of key molecular markers specific to various MN subtypes ranging from the preganglionic column (SMAD1 and ZEB2), spinal accessory column (RUNX1 and PHOX2B), and the lateral LMC (FOXP2 and ALDH1A2). Taken together, our results (summarized in Table 6) strongly indicate that in vitro derived $\mathrm{MN}$ populations are heterogeneous consisting of several functional subtypes.

\section{Discussion}

This comprehensive study documents transcriptomic and morphological changes of iPSCs as they differentiate into motor neurons (MNs) in vitro. We performed RNA-Seq meta-transcriptome analysis of human iPSC and the four cell types representing stages of spinal motor neuron differentiation (NSC, MNP, and eMN) and maturation $(\mathrm{MN})$. The results from our genomewide transcriptome study revealed basic developmental principles of in vitro neurogenesis from iPSC that have not been elucidated by previous studies while confirming the regulatory role of TGF $\beta$, Notch, and Shh signaling pathways in the formation of adult spinal motor neurons. We further corroborated the findings from the next-generation RNA-Seq analysis with RT-qPCR gene expression assays and immunohistochemistry profiles of key pluripotency and $\mathrm{MN}$ markers. Applying novel OPaver software we found strict temporal correlation between the formation of functional neuronal network connections on a MEA chip and the expression of genes involved in the regulation of $\mathrm{Ca}^{2+}$ signaling. We found that cAMP-regulated $\mathrm{Ca}^{2+}$ signaling was inhibited on gene transcript level when iPSC traversed through the neurodevelopmental stages and was reactivated in the final stage (D28) of MN maturation when we were able to detect neuronal synaptic activity via recordings of spontaneous AP firings. 
Table 6 MN specification based on $\log _{2}$-fold change in RNA-Seq transcript levels at D28 versus D0 of the differentiation protocol. The $p$-values were calculated with the R-package. Negative $\log _{2}$-fold change corresponds to gene downregulation and the positive values indicate gene activation. $\downarrow$ indicates the gene is expected to be downregulated in the MN subtype

\begin{tabular}{|c|c|c|c|}
\hline & Gene Biomarkers & Fold change & $p$-value \\
\hline \multirow[t]{4}{*}{ General motor neuron } & $H B 9$ & 4.29740102 & 0.03478379 \\
\hline & ISLI & 9.38120075 & $1.39 \mathrm{E}-11$ \\
\hline & ISL2 & -2.71940491 & 0.12115956 \\
\hline & LHX3 & 5.98518477 & 1.09E-05 \\
\hline \multirow[t]{4}{*}{ Hypaxial motor column } & ETV1 & -4.14432026 & $8.33 \mathrm{E}-05$ \\
\hline & $H B 9$ & 4.29740102 & 0.03478379 \\
\hline & ISLI & 9.38120075 & $1.39 \mathrm{E}-11$ \\
\hline & ISL2 & -2.71940491 & 0.12115956 \\
\hline \multirow[t]{4}{*}{ Pre-ganglionic column } & SMAD1 & 1.61250783 & $3.71 \mathrm{E}-10$ \\
\hline & NOS1 & -1.1688341 & 0.1409026 \\
\hline & ZEB2 & 4.94457944 & $9.71 \mathrm{E}-19$ \\
\hline & FOXP1 & 0.76366376 & 0.10008361 \\
\hline \multirow[t]{4}{*}{ Spinal accessory column } & ALCAM & 0.82274029 & 0.14709124 \\
\hline & ISLT & 9.38120075 & $1.39 \mathrm{E}-11$ \\
\hline & $R \cup N \times 1$ & 4.90888056 & $1.01 \mathrm{E}-20$ \\
\hline & PHOX2B & 9.18779756 & $1.30 \mathrm{E}-06$ \\
\hline \multirow[t]{4}{*}{ Medial LMC } & $\downarrow H B 9$ & 4.29740102 & 0.03478379 \\
\hline & ISL2 & -2.71940491 & 0.12115956 \\
\hline & FOXP2 & 5.83531355 & $9.82 \mathrm{E}-56$ \\
\hline & ALDH1A2 & 5.96267067 & $8.55 \mathrm{E}-13$ \\
\hline \multirow[t]{4}{*}{ Lateral LMC } & $\downarrow I S L 1$ & 9.38120075 & 1.39E-11 \\
\hline & ISL2 & -2.71940491 & 0.12115956 \\
\hline & FOXP2 & 5.83531355 & $9.82 \mathrm{E}-56$ \\
\hline & ALDH1A2 & 5.96267067 & $8.55 E-13$ \\
\hline \multirow[t]{4}{*}{ Median motor column } & $H B 9$ & 4.29740102 & 0.03478379 \\
\hline & ISLI & 9.38120075 & $1.39 \mathrm{E}-11$ \\
\hline & ISL2 & -2.71940491 & 0.12115956 \\
\hline & LHX3 & 5.98518477 & 1.09E-05 \\
\hline
\end{tabular}

Inhibition of $\mathrm{Ca}^{2+}$ signaling is required for the transition from iPSC to NSC

Intracellular $\mathrm{Ca}^{2+}$ levels, known as calcium transients, regulate neuronal subtype and neurotransmitter specifications [36, 37]. Our global transcriptome data showed activation of genes regulating $\mathrm{Ca}^{2+}$ entry, specifically voltage-sensitive calcium channels and ionotropic glutamate receptors, not sooner than at the eMN developmental stage (D18 vs D13, Table 1 and Fig. 5 d). $\mathrm{Ca}^{2+}$ signaling comprises a cascade of molecular interactions and biophysical events, which translate extracellular signals to intracellular responses via increase of cytoplasmic $\mathrm{Ca}^{2+}$. This can be activated by neurotransmitters, hormones, and growth factors, chemical and electrical stimuli, causing membrane excitation. Two fundamental mechanisms regulate $\mathrm{Ca}^{2+}$ entry through protein channels: voltage-dependent $\mathrm{Ca}^{2+}$ channels and ligandgated channels. The latter are highly diverse, non- $\mathrm{Ca}^{2+}$ specific, and greatly represented by the family of guanine-binding GPCR [38]. Significant upregulation in differentiated $\mathrm{MN}$ of high-voltage activated Cavs genes, such as $C A C N B 3$, is responsible for tight control on intracellular $\mathrm{Ca}^{2+}$ signaling through regulation of calcium $\left(\mathrm{Ca}^{2+}\right)$ entry and direct interaction with phospholipase C-coupled (PLC) and inositol trisphosphate (IP3) receptors [39]. Upregulation of CXCR4 by 16 -fold at D0D7 underlines the importance of intracellular $\mathrm{Ca}^{2+}$ signaling inhibition as the neuronal cell lineage commitment process begins. The CXCR4/CXCR7 complex recruits $\beta$-arrestin to trigger the canonical GPCR pathway activating ERK1/2, p38, and SAPK, while inhibiting both $\mathrm{Ca}^{2+}$ mobilization and cAMP signaling [40]. 
Upregulation of neuromedin and apelin receptors (NMUR2 and APLNR) in NSCs suggest a role for phosphoinositide (PI) signaling pathways that inhibit cAMP production upon $\mathrm{Ca}^{2+}$ mobilization and possibly in regulating cytoskeleton dynamics, cell growth, and hormone release $[41,42]$.

Our findings underline the role of temporal gene regulation of $\mathrm{Ca}^{2+}$ signaling in motor neuron development in vitro. It has been previously demonstrated that voltage-gated $\mathrm{Ca}^{2+}$ influx activated by cAMP is instrumental in the maturation of neuronal progenitor cells into functional neurons [43]. The comparative transcriptome study presented here revealed that genes regulating cAMP synthesis (ADCY2), voltage-gated calcium channels (CACNA1B and CACNG5), and the receptor regulating the intracellular $\mathrm{Ca}^{2+}$ homeostasis (RYR2) were immediately inhibited upon transition of iPSC into NSC and were re-activated in mature MN. Collectively, our data suggests that inhibition of pathways regulating the $\mathrm{Ca}^{2+}$ transients is required for the successful transition from pluripotency to neuronal progenitor cells. Our findings corroborate previous studies that have demonstrated that low cellular excitability is vital for cell migration while increase in the $\mathrm{Ca}^{2+}$ transients stops migration and promotes dendrite formation in cortical neurons [44].

\section{MN specification in vitro is driven by the chemical stimuli in growth media and bare the characteristics of the parasympathetic nervous system}

The transcriptome analysis revealed the underlying molecular changes in differentiation of iPSC to mature MNs. Specifically, we observed that short-term inhibition of TGF $\beta$ signaling was sufficient to push iPSCs into NSCs and that continuous activation of Notch and Shh signaling pathways ensured morphogenesis and cell survival throughout the MN differentiation and maturation process. While the majority of changes were characteristic of neuronal development, deeper analysis revealed the involvement of more unexpected genes as well as unique temporal changes. For example, the oscillatory pattern of SKIL gene expression throughout the neuronal cell differentiation process suggests a regulatory feed-back loop that balances survival and cell differentiation programs. PTCH1, a Shh receptor gene, is consistently and significantly upregulated from D7 to D28 suggesting a role as a positive regulator in neuronal cell division. Binding of Shh to PTCH1 results in the release of the smoothened protein initiating cell proliferation. Upon ligand binding, PTCH1 is trafficked away from the Shh positive regulator, G-coupled receptor SMO, resulting in downstream signal transduction [45]. Downregulation of PTCH1 by D28 supports the possible inhibition of cell proliferation and subsequent differentiation to neurons. ARHGAP36 and CRMP1, both of which are downregulated simultaneously, encode for Rho GTPase activating protein 36 and collapsin response mediator protein 1, are implicated in semaphorin-induced growth cone and axon guidance [46, 47] and may regulate cell division and morphogenesis of MNs.

Applying quantitative gene transcript analysis and immunohistochemistry, we observed that ChAT, a marker of mature neurons, was upregulated as soon as iPSC committed to a neuronal lineage (D7). In addition, the well-orchestrated expression of key genes such as Nestin, Pax6, NgN2, MAP2, Isl1 and HB9 corroborated the phenotypic changes to MNs. The patterns of gene expression reported here are consistent with similar studies using small molecules to drive neuron reprogramming from astrocytes [3] or fibroblasts [48].

While Shh drives motor neuron formation over intermediate neurons, there are multiple subtypes of MNs. In the final stage of $\mathrm{MN}$ maturation, we observed that genes encoding ionotropic nAChR (CHRNA3) and dopamine receptor $(D R D 2)$ were downregulated while adrenergic receptor $(A D R A 1 A)$ and $\mathrm{mAChRs}$ (CHRM2 and CHRM3) gene expression was activated. Since mAChRs are found exclusively on the neurons from the parasympathetic system [49] our data indicates that in vitro iPSC-derived MN in monoculture have the characteristics of the parasympathetic neurons of the peripheral nervous system.

In addition to the parasympathetic neuron-specific $\mathrm{mAChRs}$ gene activation, genes encoding excitatory neurotransmitters specific to the sympathetic neurons, including glutamate and catecholamines (ADRA1A), were significantly upregulated in the population of mature motor neurons. This could stem from the formation of a heterogeneous population consisting of parasympathetic and sympathetic MNs. Previous studies based on singlecell RNA-Seq analysis of iPSC-derived spinal MN have demonstrated that the protocol for in vitro differentiation produces a mixed population of $\mathrm{MN}$ subtypes with a predominant (58\%) sub-population of lateral MNs and several minority sub-populations, including hypaxial motor column (19\%) and median motor column (6\%) MNs [8].

\section{The neuroactive ligands define electrophysiological activity of in vitro iPSC-derived mature MNs}

The electrophysiological activity of mature MN reflects their ability to form functional neuronal network connections. Neuronal precursors and neurons are capable of spontaneous electrical activity [50], and we observed increased electrical activity as synapses and neural circuits formed. As iPSCs differentiated after plating on the MEAs, dendrite projections formed as axons stretched 
between neuronal cell bodies. These networks of MNs created numerous synapses to propagate nerve impulses. The MEAs were able to capture spontaneous firings of extracellular action potentials as single spikes of activity early in differentiation which progressed to more frequent firing bursts as the iPSC-derived MNs matured, demonstrating peak activity on D31. While the length and amplitude of an action potential are always the same, an increase in the stimulus caused an increase in the frequency of an action potential indicative of an enhanced response.

MNs are often characterized by their role in forming cholinergic synapses at NMJs. MN axons terminate on muscle fibers and nerve impulses are translated into muscle contractions as the neurotransmitter ACh is released from the presynaptic $\mathrm{MN}$ terminal for uptake by postsynaptic ACh receptors on the target muscle cell [1]. Understanding the underlying mechanism of cholinergic development provides insights about potential treatments for neurodegenerative diseases and strategies to develop countermeasures to chemical toxicology exposure. AChE inhibitors are used to treat Alzheimer's and Parkinson's diseases [51, 52] and cholinergic agonist treatments have shown to improve memory function [53]. Similar strategies have been employed to treat organophosphate-induced neurotoxicity [54, 55]. Here we show how culture conditions imprint distinct fates and future efforts to co-culture of MNs with other cell types, such as muscle, may cause different neuronal specification. Surprisingly, we observed the expression of nAChRs typically found on the NMJ to be downregulated in mature $\mathrm{MN}$ monocultures while the mAChRs were upregulated. These results can further be applied to characterize aberrant neuronal functions following neurodegeneration or exposure to chemical toxins.

\section{Conclusions}

This study revealed significant changes to the transcriptome, protein expression, and electrical function as iPSCs differentiated into mature MNs. To ensure cellular mobility that is essential for tissue layer formation, genes regulating $\mathrm{Ca}^{2+}$ signaling are downregulated throughout the cell differentiation process and are activated in mature MN. Differentiation of iPSC into MN in vitro monocultures leads to formation of both parasympathetic and sympathetic neuronal types. Downregulation of tumor suppressing genes upon chemical conversion of iPSC to mature MN underlines the danger from direct application of the technology in vivo. Understanding the underlying molecular and cellular cues involved in MN differentiation of iPSCs has the potential to enable the discovery of novel treatments for neural injuries.

\section{Methods}

\section{Culturing and differentiating iPSCs}

Human iPSCs (WTC-11, Coriell Institute) were cultured and maintained on vitronectin (ThermoFisher Scientific) treated culture plates in Essential8 medium (ThermoFisher Scientific). Differentiation of iPSCs into MNs was directed as previously described [6] with slight modifications that substituted the NSC growth in suspension (D7-D13) with a growth on vitronectin substrate to improve the cell survival rate and to ensure gene expression profile characteristic to motor neuron progenitor cells (MNP). Briefly, iPSCs were cultured in neural media which consisted of 1:1 DMEM/F12 and Neurobasal medium supplemented with N2, B27, 1x Glutamax and $1 \mathrm{x}$ penicillin/streptomycin (all from ThermoFisher Scientific), and $0.1 \mathrm{mM}$ ascorbic acid (StemCell Technology). On days 0-6 $3 \mu \mathrm{M}$ CHIR99021 (StemCell Technology), $2 \mu \mathrm{M}$ DMH-1 (Tocris) and $2 \mu \mathrm{M}$ SB431542 (StemCell Technology) were added to the neural medium; days 6-12 the same media was used with the addition of $0.1 \mu \mathrm{M}$ RA (StemCell Technology) and $0.5 \mu \mathrm{M}$ Pur (Sigma); days $12-18$ cells were maintained with $0.1 \mu \mathrm{M}$ RA and $0.5 \mu \mathrm{M}$ Pur added to the neural media; finally from day 18 on cells were cultured with $0.5 \mu \mathrm{M}$ RA, $0.1 \mu \mathrm{M}$ Pur and $0.1 \mu \mathrm{M}$ CpdE (StemCell Technology), IGF-1, BDNF, and CNTF (all from R\&D Systems, $10 \mathrm{ng} / \mathrm{ml}$ each). For optimum neuronal network formation, cells at the MNP differentiation stage (D12) were plated on MEA at $60 \%$ area density.

\section{RNA extraction}

Cells were lifted with accutase, pelleted by centrifugation, and stored at $-20^{\circ} \mathrm{C}$. Total RNA was extracted from cell pellets using RNeasy Mini Kit (Qiagen), following the recommendations of the manufacturer. After DNase digestion by Turbo DNA-free kit (ThermoFisher Scientific), samples were quantified and divided for qPCR and transcriptomic analyses.

\section{RNA sequencing analysis}

Extracted and DNase-treated RNA was quantified using the Qubit 4 Fluorometer (ThermoFisher) with the High Sensitivity RNA reagents and Bioanalyzer (Agilent) with RNA 6000 Pico reagents. Ribosomal depletion, DNA conversion, and library preparation was performed on all samples using the Illumina TruSeq Stranded Total RNA kit. 151 base pair reads were sequenced on the Illumina NextSeq. Across fifteen samples (three independent experiments $x$ five time points) the total number of reads generated for each sample ranged from approximately 26 million to 40 million reads. Sequencing data was quality trimmed using FaQCs [56] with a quality score cutoff of Q20. Differential expression analysis was performed using PiReT [57] V 0.3.2 and utilizing DEseq2 
[58] default parameters and setting a q-value of 0.05 (false discovery rate metric). The experimental design file (provided in the supplementary material) was used to dictate the replicate sample ID's and sequencing data to be used in the PiReT analysis. Human genome version hg38 was used as the reference genome. KEGG [59, 60] pathway mapping was performed using Omics Pathway Viewer - 'OPaver' (Li, unpublished). Raw RNA-Seq reads were deposited in the NCBI SRA database under the accession numbers SRR11994167- SRR11994181. Metadata for each sample are also accessible under NCBI BioProject PRJNA638768.

\section{Quantitative reverse transcription PCR (RT-qPCR)}

Three independent experiments were run in duplicate using a 7500 Fast Real-Time PCR System (Applied Biosciences). Fifty ng of each RNA sample were probed for motor neuron differentiation markers using Taqman RNA-to-CT 1-Step Kit (Applied Bioscience) in a $25 \mu \mathrm{l}$ volume according to the manufacturer's instructions. Taqman probes included NEUROG2 (Hs00935087_g1), ChAT (Hs00758143_m1), Isl1 (Hs01099686_m1), PAX6 (Hs01088114_m1), MAP2 (Hs00258900_m1), Nestin (Hs04187831_g1), Oct4 (Hs00999632_g1), and HB9 (Hs00907365_m1). Two endogenous controls, actin (Hs99999903_m1) and GAPDH (Hs01922876_u1), were analyzed by RT-qPCR and no significant differences were observed in their expression levels (data not shown); thus, all data shown are normalized to GAPDH.

\section{Immunocytochemistry staining and analysis}

Nunc Lab-Tek chamber slides (ThermoFisher Scientific) were coated with vitronectin, seeded, and subsequently fixed with $4 \%$ paraformaldehyde, permeabilized with $0.4 \%$ Triton X-100, blocked with 3\% BSA in PBS for at least 1 h. Samples were incubated overnight at $4{ }^{\circ} \mathrm{C}$ with primary antibody solutions (Table 6) diluted in PBS containing Image-iT FX signal enhancer. Cells were washed with PBS three times prior to incubation with NucBlue Fixed Cell
Reagent, Image-iT FX signal enhancer, and secondary Alexa-488-, Alexa 555-, or Alexa 647-conjugated antibodies at $37^{\circ} \mathrm{C}$ for $2 \mathrm{~h}$ (1:1000 dilution, ThermoFisher Scientific and Jackson ImmunoResearch). Table 7 summarizes the antibodies and their concentrations applied in this study. After three PBS washes, the media chambers were removed from the glass slide, coverslips were mounted using ProLong Diamond Antifade Mountant and cells were examined using fluorescence microscopy (Zeiss Observer Z.1). For biomarker quantification, images were acquired with an AxioCam camera connected to Axio Observer Z1 microscope, using ZEN software. For each culture, images were taken with a $20 \mathrm{X}$ objective choosing fields with $>100$ cells. Images from 5 random fields per culture condition were analyzed with Image J with the following parameters: (i) manual threshold, (ii) fill holes, (iii) enhance contrast and saturated pixels at $0.3 \%$, (iv) watershed separation, and (v) particle analysis at size 100 -infinity and circularity at $0.2-0.1$. The fraction of cells expressing biomarker proteins was calculated as percent of total cells labeled with DAPI.

Functional analysis of MNs on microelectrode array (MEA) Cells were seeded on MEA chips, either in 60MEA200/ 30iR-Ti arrays or 24-well Plate with PEDOT Electrodes on Glass, 24 W300/30G-288 (Multichannel Systems). MEA's were coated with poly-D-lysine and vitronectin. Before recording, the MEA chips were moved to the MEA2100 system (MultiChannel Systems) equipped with temperature control and allowed to equilibrate for $10 \mathrm{~min}$ before recording. The data were acquired using Multi Channel Experimenter or Multiwell Screen (MultiChannel Systems) at a sampling rate of $20 \mathrm{kHz}$ for $2 \mathrm{~min}$ at $37^{\circ} \mathrm{C}$. Data were filtered using Butterworth band pass filter with $200 \mathrm{~Hz}$ cutoff frequency and threshold of $5 \times$ SD were set to minimize both false-positive and missed detection. The representative electrodes were selected for analysis of mean spike frequency and percentage of spikes in the burst.

Table 7 List of primary antibodies

\begin{tabular}{llll}
\hline Antibody & Species & Dilution & Source \\
\hline Oct4 & Rabbit & $1: 200$ & Cell Signaling Technology (Cat\# 2750S) \\
Nestin & Rabbit & $1: 200$ & Abcam (Cat\# ab105389) \\
Pax6 & Mouse & $1: 50$ & Developmental Studies Hybridoma Bank (Cat\# Pax6) \\
MAP2 & Mouse & $1: 200$ & ThermoFisher (Cat\# MA5-12823) \\
HB9 / MNX1 & Mouse & $1: 50$ & Developmental Studies Hybridoma Bank (Cat\# 81.5C10) \\
ChAT & Goat & $1: 100$ & Milipore (Cat\#AB144P) \\
beta-III-tubulin & Rabbit & $1: 1000$ & Abcam (Cat\# ab18207) \\
Synaptophysin [SY38] & Mouse & $1: 100$ & Abcam (Cat\# ab8049) \\
\hline
\end{tabular}




\section{Transcriptome analysis}

DAVID Functional Annotation Bioinformatics Microarray Analysis can be accessed at https://david.ncifcrf.gov. The raw transcriptomic data of D0-D28 significant DEGs $(p<0.05)$ included 2242 upregulated and 1438 downregulated terms, available in the supplemental material. Each list of Homo sapiens genes was independently analyzed by DAVID, to generate an analysis of associated gene ontology $(\mathrm{GO})$ terms.

OPaver ( $\mathrm{Li}$, unpublished), is a web-based tool to integrate multiple types (e.g. transcriptomics, proteomics and metabolomics) and time series of data to KEGG biochemical pathways maps. This software analysis tool was developed at Los Alamos National Laboratory. In this case, OPaver was utilized to map significantly differentially expressed genes $(p<0.05)$ identified in the DEseq2 analysis performed by PiRet. Differential expression calculation from DEseq2 in Log2 fold change and associated genes (provided in the supplementary material) were used as input for the OPaver software.

\section{Supplementary Information}

The online version contains supplementary material available at https://doi. org/10.1186/s12860-021-00343-z.

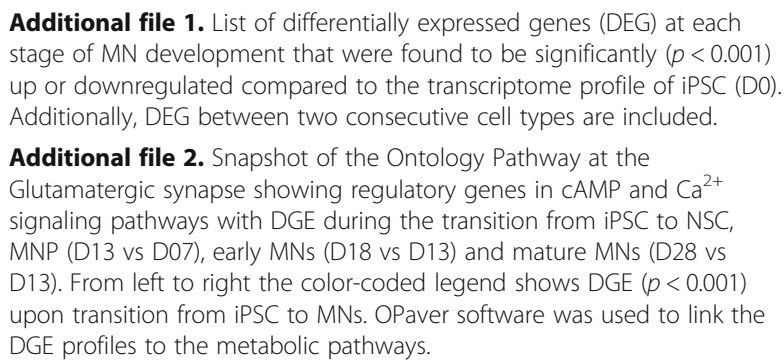

\begin{abstract}
Abbreviations
ACh: Acetylcholine; ALS: Amyotrophic lateral sclerosis; AP: Action potentials; BDNF: Brain-derived neurotrophic factor; $\beta$ III-Tub: Class III $\beta$-tubulin; BMP: Bone morphogenic protein; CAMP: cyclic adenosine monophosphate; Cavs: Voltage-dependent calcium-channels; ChAT: Choline acetyltransferase; CHIR: CHIR99021; CNTF: Ciliary neurotrophic factor; CpdE: Compound E; D0: Day 0; D7: Day 7; D13: Day 13; D18: Day 18; D28: Day 28; D31: Day 31; DAVID: Database for annotation, visualization and integrated discovery; DEGs: Differentially expressed genes; eMNs: Early motor neurons; GO: Gene ontology; GPCR: G protein-coupled receptors; HVA: High-voltage activated; IP3: Inositol trisphosphate; iPSC: Induced pluripotent stem cells; IGF-1: Insulinlike growth factor 1; IsI1: Islet-1; LVA: Low-voltage activated; LANL: Los Alamos national laboratory; mAChRs: Muscarinic acetylcholine receptors; MAP 2: Microtubule-associated protein 2; MEA: Multi-electrode array; MN: Motor neurons; MNPs: Motor neuron progenitors; nAChR: Nicotinic acetylcholine receptor; NMJs: Neuromuscular junctions; NSCs: Neural stem cells; OPaver: Ontology pathway analysis software; PCA: Principal component analysis; PLC: Phospholipase C; Pur: Purmorphamine; RA: All-trans retinoic acid; RNA-Seq: RNA-sequencing; SB: SB431542; Shh: Sonic hedgehog.; TGF $\beta$ : Tumor growth factor- $\beta$
\end{abstract}

\section{Acknowledgements}

We greatly valued helpful discussions with Paul Li and Bin Hu, as well as Arasely Rodriguez's technical assistance.

\section{Authors' contributions}

ES: Methodology, Investigation, Visualization, Formal analysis, Writing review and editing; KDA: Writing original draft, Visualization, Formal analysis; BH: Formal analysis; SMV: Formal analysis, Writing-original draft; JFH: Supervision; ST: Writing - review and editing, Supervision, and RI: Supervision, Writing - review and editing. The final version was approved by all authors.

\section{Funding}

Research presented in this article was supported by the Laboratory Directed Research and Development program of Los Alamos National Laboratory under project number 20190167.

\section{Availability of data and materials}

The datasets generated for this study can be found in the NCBI SRA database under accession numbers SRR11994167- SRR11994181, NBCI BioProject PRJNA638768, [https://www.ncbi.nlm.nih.gov/bioproject/PRJNA638768] and NCBI BioSamples SAMN15207814-SAMN15207828.

Ethics approval and consent to participate

Not applicable.

\section{Consent for publication}

Not applicable.

\section{Competing interests}

The authors declare that they have no competing interests.

Received: 28 October 2020 Accepted: 11 January 2021

Published online: 18 February 2021

\section{References}

1. Legay C, Mei L. Moving forward with the neuromuscular junction. J Neurochem. 2017;142(Suppl 2):59-63.

2. Sances S, Bruijn LI, Chandran S, Eggan K, Ho R, Klim JR, et al. Modeling ALS with motor neurons derived from human induced pluripotent stem cells. Nat Neurosci. 2016;19(4):542-53.

3. Ma NX, Yin JC, Chen G. Transcriptome analysis of small molecule-mediated astrocyte-to-neuron reprogramming. Front Cell Dev Biol. 2019;7:82.

4. Davis-Dusenbery BN, Williams LA, Klim JR, Eggan K. How to make spinal motor neurons. Development. 2014;141(3):491-501.

5. Amoroso MW, Croft GF, Williams DJ, O'Keeffe S, Carrasco MA, Davis AR, et al. Accelerated high-yield generation of limb-innervating motor neurons from human stem cells. J Neurosci. 2013:33(2):574-86.

6. Du ZW, Chen H, Liu H, Lu J, Qian K, Huang CL, et al. Generation and expansion of highly pure motor neuron progenitors from human pluripotent stem cells. Nat Commun. 2015;6:6626.

7. Petros TJ, Tyson JA, Anderson SA. Pluripotent stem cells for the study of CNS development. Front Mol Neurosci. 2011;4:30.

8. Thiry L, Hamel R, Pluchino S, Durcan T, Stifani S. Characterization of Human iPSC-derived Spinal Motor Neurons by Single-cell RNA Sequencing. Neuroscience. 2020;450:57-70. https://doi.org/10.1016/j.neuroscience.2020. 04.041 .

9. Namboori SC, Thomas P, Ames R, Garrett LO, Willis CR, Stanton LW, Bhinge A. Single cell transcriptomics identifies master regulators of dysfunctional pathways in SOD1 ALS motor neurons. bioRxiv. 2019:593129. https://doi. org/10.1101/593129.

10. Sagner A, Gaber ZB, Delile J, Kong JH, Rousso DL, Pearson CA, et al. Olig2 and Hes regulatory dynamics during motor neuron differentiation revealed by single cell transcriptomics. PLoS Biol. 2018;16(2):e2003127.

11. Maciel R, Bis DM, Rebelo AP, Saghira C, Zuchner S, Saporta MA. The human motor neuron axonal transcriptome is enriched for transcripts related to mitochondrial function and microtubule-based axonal transport. Exp Neurol. 2018:307:155-63.

12. Burke EE, Chenoweth JG, Shin JH, Collado-Torres L, Kim SK, Micali N, et al. Dissecting transcriptomic signatures of neuronal differentiation and maturation using iPSCs. Nat Commun. 2020;11(1):462.

13. Courtot AM, Magniez A, Oudrhiri N, Feraud O, Bacci J, Gobbo E, et al. Morphological analysis of human induced pluripotent stem cells during induced differentiation and reverse programming. Biores Open Access. 2014;3(5):206-16. 
14. Bott CJ, Johnson CG, Yap CC, Dwyer ND, Litwa KA, Winckler B. Nestin in immature embryonic neurons affects axon growth cone morphology and Semaphorin3a sensitivity. Mol Biol Cell. 2019;30(10):1214-29.

15. Sunabori T, Tokunaga A, Nagai T, Sawamoto K, Okabe M, Miyawaki A, et al. Cell-cycle-specific nestin expression coordinates with morphological changes in embryonic cortical neural progenitors. J Cell Sci. 2008;121(Pt 8):1204-12.

16. Arber S, Han B, Mendelsohn M, Smith M, Jessell TM, Sockanathan S. Requirement for the homeobox gene $\mathrm{Hb}$ in the consolidation of motor neuron identity. Neuron. 1999;23(4):659-74.

17. Harada A, Teng J, Takei Y, Oguchi K, Hirokawa N. MAP2 is required for dendrite elongation, PKA anchoring in dendrites, and proper PKA signal transduction. J Cell Biol. 2002;158(3):541-9.

18. Soltani MH, Pichardo R, Song Z, Sangha N, Camacho F, Satyamoorthy K, Sangueza OP, Setaluri V. Microtubule-associated protein 2, a marker of neuronal differentiation, induces mitotic defects, inhibits growth of melanoma cells, and predicts metastatic potential of cutaneous melanoma. Am J Patho. 2005;166(6):1841-50.

19. Huang DW, Sherman BTL, R.A. Systematic and integrative analysis of large gene lists using DAVID bioinformatics resources. Nat Protoc. 2009;4(1):44-57.

20. Li PECP. OPaver: Omics Pathway Viewer.

21. Tecalco-Cruz AC, Sosa-Garrocho M, Vazquez-Victorio G, Ortiz-Garcia L, Dominguez-Huttinger E, Macias-Silva M. Transforming growth factorbeta/SMAD target gene SKIL is negatively regulated by the transcriptional cofactor complex SNON-SMAD4. J Biol Chem. 2012; 287(32):26764-76

22. Huang P, Xiong F, Megason SG, Schier AF. Attenuation of notch and hedgehog signaling is required for fate specification in the spinal cord. PLOS Genet. 2012;8(6):e1002762.

23. Fukunaga K, Miyamoto E. Role of MAP kinase in neurons. Mol Neurobiol. 1998;16(1):79-95.

24. Lacinova L. Voltage-dependent calcium channels. Gen Physiol Biophys. 2005;24(Suppl 1):1-78.

25. Brini M, Cali T, Ottolini D, Carafoli E. Neuronal calcium signaling: function and dysfunction. Cell Mol Life Sci. 2014;71(15):2787-814.

26. Tan W, Dean M, Law AJ. Molecular cloning and characterization of the human ErbB4 gene: identification of novel splice isoforms in the developing and adult brain. PLoS One. 2010;5(9):e12924.

27. Chung DW, Wills ZP, Fish KN, Lewis DA. Developmental pruning of excitatory synaptic inputs to parvalbumin interneurons in monkey prefrontal cortex. Proc Natl Acad Sci U S A. 2017;114(4):E629-E37.

28. Murakami M, Ohba T, Xu F, Satoh E, Miyoshi I, Suzuki T, et al. Modified sympathetic nerve system activity with overexpression of the voltagedependent calcium channel beta3 subunit. J Biol Chem. 2008;283(36): 24554-60.

29. Takahashi K, Tanaka A, Hara M, Nakanishi S. The primary structure and gene organization of human substance $P$ and neuromedin $K$ receptors. Eur J Biochem. 1992;204(3):1025-33.

30. Steinhoff MS, von Mentzer B, Geppetti P, Pothoulakis C, Bunnett NW. Tachykinins and their receptors: contributions to physiological control and the mechanisms of disease. Physiol Rev. 2014;94(1):265-301.

31. Watson LM, Bamber E, Schnekenberg RP, Williams J, Bettencourt C, Lickiss J, et al. Dominant mutations in GRM1 cause Spinocerebellar Ataxia type 44. Am J Hum Genet. 2017;101(5):866.

32. Scott GD, Fryer AD. Role of parasympathetic nerves and muscarinic receptors in allergy and asthma. Chem Immunol Allergy. 2012;98:48-69.

33. Arikawa K, Takuwa N, Yamaguchi H, Sugimoto N, Kitayama J, Nagawa H, et al. Ligand-dependent inhibition of B16 melanoma cell migration and invasion via endogenous S1P2 G protein-coupled receptor. Requirement of inhibition of cellular RAC activity. J Biol Chem. 2003;278(35):32841-51.

34. Lukomska A, Dobrzanski G, Liguz-Lecznar M, Kossut M. Somatostatin receptors (SSTR1-5) on inhibitory interneurons in the barrel cortex. Brain Struct Funct. 2020;225(1):387-401.

35. Santiago C, Labrador JP, Bashaw GJ. The homeodomain transcription factor $\mathrm{Hb}$ 9 controls axon guidance in Drosophila through the regulation of Robo receptors. Cell Rep. 2014;7(1):153-65.

36. Moore AR, Filipovic R, Mo Z, Rasband MN, Zecevic N, Antic SD. Electrical excitability of early neurons in the human cerebral cortex during the second trimester of gestation. Cereb Cortex. 2009;19(8):1795-805.

37. Moore AR, Zhou WL, Jakovcevski I, Zecevic N, Antic SD. Spontaneous electrical activity in the human fetal cortex in vitro. J Neurosci. 2011; 31(7):2391-8.
38. Birnbaumer L, Zhu X, Jiang M, Boulay G, Peyton M, Vannier B, et al. On the molecular basis and regulation of cellular capacitative calcium entry: roles for Trp proteins. Proc Natl Acad Sci. 1996;93(26):15195-202.

39. Belkacemi A, Hui X, Wardas B, Laschke MW, Wissenbach U, Menger MD, et al. IP3 receptor-dependent cytoplasmic $\mathrm{Ca}(2+)$ signals are tightly controlled by Cavbeta3. Cell Rep. 2018;22(5):1339-49.

40. Decaillot FM, Kazmi MA, Lin Y, Ray-Saha S, Sakmar TP, Sachdev P. CXCR7/ CXCR4 heterodimer constitutively recruits beta-arrestin to enhance cell migration. J Biol Chem. 2011;286(37):32188-97.

41. Chapman NA, Dupre DJ, Rainey JK. The apelin receptor: physiology, pathology, cell signalling, and ligand modulation of a peptide-activated class A GPCR. Biochem Cell Biol. 2014;92(6):431-40.

42. Brighton PJ, Szekeres PG, Willars GB. Neuromedin U and its receptors: structure, function, and physiological roles. Pharmacol Rev. 2004;56(2):231-48.

43. Lepski G, Jannes C, Nikkhah G, Bischofberger J. cAMP promotes the differentiation of neural progenitor cells in vitro via modulation of voltagegated calcium channels. Front Cell Neurosci. 2013;7(155):1.

44. Bando $Y$, Irie K, Shimomura T, Umeshima H, Kushida Y, Kengaku M, et al. Control of spontaneous Ca2+ transients is critical for neuronal maturation in the developing Neocortex. Cereb Cortex. 2016;26(1):106-17.

45. Dessaud E, MCMahon AP, Briscoe J. Pattern formation in the vertebrate neural tube: a sonic hedgehog morphogen-regulated transcriptional network. Development. 2008;135(15):2489-503.

46. Nam H, Jeon S, An H, Yoo J, Lee HJ, Lee SK, et al. Critical roles of ARHGAP36 as a signal transduction mediator of Shh pathway in lateral motor columnar specification. Elife. 2019:8:e46683.

47. Nakamura F, Kumeta K, Hida T, Isono T, Nakayama Y, Kuramata-Matsuoka E, et al. Amino- and carboxyl-terminal domains of Filamin-A interact with CRMP1 to mediate Sema3A signalling. Nat Commun. 2014;5:5325.

48. Dimos JT, Rodolfa KT, Niakan KK, Weisenthal LM, Mitsumoto H, Chung W, Croft GF, Saphier G, Leibel R, Goland R, Wichterle H, Henderson CE, Eggan K. Induced pluripotent stem cells generated from patients with ALS can be differentiated into motor neurons. Science. 2008:321(5893):1218-21.

49. McCorry LK. Physiology of the autonomic nervous system. Am J Pharm Educ. 2007:71(4):78.

50. Blankenship AG, Feller MB. Mechanisms underlying spontaneous patterned activity in developing neural circuits. Nat Rev Neurosci. 2010;11(1):18-29.

51. McGleenon BM, Dynan KB, Passmore AP. Acetylcholinesterase inhibitors in Alzheimer's disease. Br J Clin Pharmacol. 1999;48(4):471-80.

52. Pagano G, Rengo G, Pasqualetti G, Femminella GD, Monzani F, Ferrara N, et al. Cholinesterase inhibitors for Parkinson's disease: a systematic review and meta-analysis. J Neurol Neurosurg Psychiatry. 2015;86(7):767-73.

53. Hampel H, Mesulam MM, Cuello AC, Farlow MR, Giacobini E, Grossberg GT, et al. The cholinergic system in the pathophysiology and treatment of Alzheimer's disease. Brain. 2018;141(7):1917-33.

54. Kaur S, Singh S, Chahal KS, Prakash A. Potential pharmacological strategies for the improved treatment of organophosphate-induced neurotoxicity. Can J Physiol Pharmacol. 2014:92(11):893-911.

55. Colovic MB, Krstic DZ, Lazarevic-Pasti TD, Bondzic AM, Vasic VM. Acetylcholinesterase inhibitors: pharmacology and toxicology. Curr Neuropharmacol. 2013;11(3):315-35.

56. Lo CC, Chain PS. Rapid evaluation and quality control of next generation sequencing data with FaQCs. BMC Bioinformatics. 2014;15:366.

57. Shakya MC, P. PiReT Pipeline for Reference based Transcriptomics.

58. Love MI, Huber W, Anders S. Moderated estimation of fold change and dispersion for RNA-seq data with DESeq2. Genome Biol. 2014;15(12):550.

59. Kanehisa M. Toward understanding the origin and evolution of cellular organisms. Protein Sci. 2019;28(11):1947-51.

60. Kanehisa M, Goto S. KEGG: Kyoto encyclopedia of genes and genomes. Nucleic Acids Res. 2000;28(1):27-30

\section{Publisher's Note}

Springer Nature remains neutral with regard to jurisdictional claims in published maps and institutional affiliations. 